\title{
THE EFFECT OF REAPING TIMES ON VOLATILE COMPONENTS OF NATURAL PHLOMIS L. (LAMIACEAE) TAXA IN THE LAKES DISTRICT OF TURKEY
}

\author{
SARIKAYA, A. G. ${ }^{*}-$ FAKIR, H. $^{2}$ \\ ${ }^{1}$ Suleyman Demirel University, Atabey Vocational School, Isparta-Turkey \\ ${ }^{2}$ Suleyman Demirel University, Faculty of Forestry, Forest Engineering Department, Isparta- \\ Turkey \\ *Corresponding author \\ e-mail: aysegulsarikaya@sdu.edu.tr \\ (Received $14^{\text {th }}$ Feb 2016; accepted $23^{\text {rd }}$ Jun 2016)
}

\begin{abstract}
This study performed SPME analysis in the Lakes District of Turkey between 2012-2015 and found that Phlomis armeniaca includes 54 volatile components, P. bourgaei 62, P. grandiflora var. grandiflora 60, P. leucophracta 70, P. lycia 62, P. nissolii' 54, P. pungens var. pungens 74 and $P$. samia 49. The main components were (E)-2-hexenal $(12.12 \%), \beta$-caryophyllene $(16.63 \%)$ and germacrene-D (27.22\%) for P. armeniaca; $\alpha$-cubebene (16.04\%), $\beta$-caryophyllene (21.98\%) and germakren-D (15.12\%) for P. bourgaei; $\alpha$-pinene (26.40\%), $\alpha$-cedrene (28.15\%) and $\alpha$-curcumene (13.92\%) for P. grandiflora var. grandiflora; (E)-2-hexenal (8.74\%), limonene (14.56\%) and $\beta$-caryophyllene $(22.45 \%)$ for $P$. leucophracta, limonene (17.68\%), $\beta$-caryophyllene (23.66\%) and germacrene-D (21.88\%) for P. lycia; limonene (23.75\%), $\beta$-caryophyllene (12.50\%) and germacrene-D (20.73\%) for Phlomis nissolii; (E)-2hexenal (17.60\%), vinly amyl carbinol (20.44\%) and germacrene-D $(9.84 \%)$ for $P$. pungens and $\alpha .-$ copaene (10.59\%), $\beta$-caryophyllene (15.20\%) and germacrene-D (23.44\%) for P. samia.
\end{abstract}

Keywords: Phlomis, volatile component, SPME analyses, $\beta$-caryophyllene, germakren-D, Turkey

\section{Introduction}

The flora in Turkey has approximately 11.466 plant taxa. As a comparison, the European continent has approximately 12.000 (Guner et al., 2012). Turkey also has species variety that is the gene center of several plants and there are many endemic species in different geographical regions (Tan, 1992). In particular for the endemics, aromatic and medical values of hundreds of plant types grown in Turkey are naturally higher (Baydar, 2009). This is especially the case for volatile oil content in medicinal and aromatic plant groups which has a separate importance. Volatile oils (perfumes, etheric oils) and aromatic extracts are commonly used for perfume production, to enhance smell and taste, as food additives, in cleaning products, in cosmetic and drugs, and as sources of aroma chemicals or identical natural and semi-synthetic aroma chemicals for the synthesis of starting materials. Currently, there is increasing demand for volatile oils in aromatherapy applications (Weiss, 1997).

The Lamiaceae family has generally sweet smelling one or multi perennial plants that are rarely briers and with some trees. This cosmopolite family is represented by 200 genera and approximately 3000 species. Lamiaceae (labiatate) family members that are represented by 45 genera and 546 species in Turkey are important for the pharmacology and perfumery industry due to their volatile and aromatic oils. Etheric oil is an example that is used as a spice is also grown as a decorative plant. The Phlomis taxa which has the most species of genera of the Lamiaceae family, has over 100 species all around the world. The taxa of this genus are 
distributed in Asia, South Europe and North Europe (Matthiesen et al., 2011). In Turkey, it is represented by 39 taxon and 13 hybrids, for a total of 52 taxon (Guner et al., 2012).

The discovery of new usage areas for medical and aromatic plants, increasing demand for natural products, increases the demand for these plants each passing day. The medical plant market is estimated to currently reach approximately 60 billion dollars (Kumar, 2009). Moreover, there is growing public interest towards such plants and for use of aromatic and medical plants.

Phlomis taxa have an important place in the natural distribution of medical plants and one of the species that has the most types of the Lamiaceae family. It has about 100 species all around the world. The length of species can vary between $30 \mathrm{~cm}$ and 2 meters. The sides of leaves are jagged and opposing in alignment but are not in a bulk condition. Feathers covering the surface of plant are stellate. Flowers are purple, pink, white or yellow colored (HuberMorath, 1982). Leaves and flowers are used to make products more appetizing, as an antiallergic, as a diuretic, for diarrhea prevention, against stomach aches, to relieve pain, as an anti-diabetic, herbal tea and tonic. The plant is also colloquially known to be used for respiratory tract diseases and hemorrhoid problems (Harput et al., 2006).

Although it is used in many areas, there is little research about the Phlomis taxa of Turkey. Research about the volatile oil of leaves is also limited. For these reasons, this study aimed to research the effect of different picking periods in terms of volatile oil compounds and to determine suitable picking periods for Phlomis armeniaca Willd., P. bourgaei Boiss., $P$. grandiflora H.S. Thompson var. grandiflora, P. leucophracta P.H.Davis \& Hub.-Mor., $P$. lycia D. Don., P. nissolii L., P. pungens Willd. and P. samia L.

\section{Materials and Methods}

Phlomis samples were collected from the Lakes District of Turkey (C2, C3, C4 squares) between the years 2012 and 2015 (Figure 1). Thirty-three samples for Phlomis species were collected from the Lakes District in three different annual time periods: pre-bloom period, bloom period, and post-emergence period (Table 1).

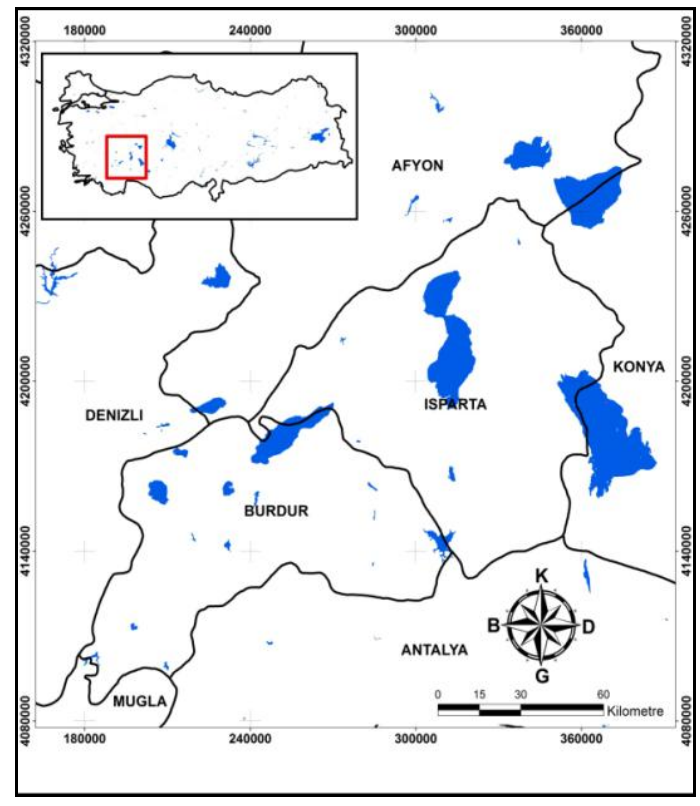

Figure 1. Research area 
Table 1. Ecological land informations of collecting samples

\begin{tabular}{|c|c|c|c|}
\hline Taxa & Province & Location & Altitude (m) \\
\hline \multirow[t]{6}{*}{$\begin{array}{l}\text { Phlomis armeniaca } \\
\text { Willd. }\end{array}$} & Afyon-Çay county & $\begin{array}{l}37^{\circ} 47^{\prime} 08^{\prime \prime} \mathrm{N} \\
30^{\circ} 45^{\prime} 54^{\prime \prime} \mathrm{E}\end{array}$ & 830 \\
\hline & $\begin{array}{l}\text { Konya-Beyşehir county, } \\
\text { Kurucuova province }\end{array}$ & $\begin{array}{l}37^{\circ} 50^{\prime} 25^{\prime \prime} \mathrm{N} \\
31^{\circ} 06^{\prime} 11^{\prime \prime} \mathrm{E} \\
\end{array}$ & 1155 \\
\hline & $\begin{array}{l}\text { Isparta-Aksu county, } \\
\text { Sorgun Yaylas1 } \\
\text { province }\end{array}$ & $\begin{array}{l}37^{\circ} 42^{\prime} 11^{\prime \prime} \mathrm{N} \\
31^{\circ} 17^{\prime} 38^{\prime \prime} \mathrm{E}\end{array}$ & 1490 \\
\hline & $\begin{array}{l}\text { Isparta- Davraz } \\
\text { mountain, Ardıçdibi } \\
\text { province }\end{array}$ & $\begin{array}{l}37^{\circ} 48^{\prime} 09^{\prime \prime} \mathrm{N} \\
30^{\circ} 45^{\prime} 28^{\prime \prime} \mathrm{E}\end{array}$ & 1604 \\
\hline & $\begin{array}{l}\text { Isparta-Davraz } \\
\text { mountain }\end{array}$ & $\begin{array}{l}37^{\circ} 41^{\prime} 55^{\prime \prime} \mathrm{N} \\
31^{\circ} 24^{\prime} 18^{\prime \prime} \mathrm{E}\end{array}$ & 1622 \\
\hline & $\begin{array}{l}\text { Isparta-Yenişarbademli } \\
\text { county, Melikler } \\
\text { Yaylası province }\end{array}$ & $\begin{array}{l}38^{\circ} 57^{\prime} 02^{\prime \prime} \mathrm{N} \\
31^{\circ} 02^{\prime} 19^{\prime \prime} \mathrm{E}\end{array}$ & 1762 \\
\hline \multirow[t]{6}{*}{ Phlomis bourgaei Boiss. } & $\begin{array}{l}\text { Isparta- Siğla } \\
\text { (Liquidambar } \\
\text { orientalis) Forest Nature } \\
\text { Protected Area }\end{array}$ & $\begin{array}{l}37^{\circ} 34^{\prime} 15^{\prime \prime} \mathrm{N} \\
30^{\circ} 50^{\prime} 06^{\prime \prime} \mathrm{E}\end{array}$ & 379 \\
\hline & $\begin{array}{l}\text { Isparta- Eğirdir county, } \\
\text { Așağıgökdere province }\end{array}$ & $\begin{array}{l}37^{\circ} 37^{\prime} 33^{\prime \prime} \mathrm{N} \\
30^{\circ} 43^{\prime} 50^{\prime \prime} \mathrm{E}\end{array}$ & 471 \\
\hline & $\begin{array}{l}\text { Antalya-Isparta } \\
\text { motoway, Dereboğazı } \\
\text { province }\end{array}$ & $\begin{array}{l}37^{\circ} 19^{\prime} 48^{\prime \prime} \mathrm{N} \\
30^{\circ} 37^{\prime} 22^{\prime \prime} \mathrm{E}\end{array}$ & 590 \\
\hline & $\begin{array}{l}\text { Isparta- Eğirdir county, } \\
\text { Aşağigökdere Akbelenli } \\
\text { province }\end{array}$ & $\begin{array}{l}37^{\circ} 33^{\prime} 57^{\prime \prime} \mathrm{N} \\
30^{\circ} 51^{\prime} 58^{\prime \prime} \mathrm{E}\end{array}$ & 705 \\
\hline & $\begin{array}{l}\text { Burdur- Karlık Kavaklık } \\
\text { province }\end{array}$ & $\begin{array}{l}37^{\circ} 44^{\prime} 28^{\prime \prime} \mathrm{N} \\
30^{\circ} 33^{\prime} 18^{\prime \prime} \mathrm{E}\end{array}$ & 1228 \\
\hline & $\begin{array}{l}\text { Isparta- Urban forest, } \\
\text { Sidre province }\end{array}$ & $\begin{array}{l}37^{\circ} 22^{\prime} 31^{\prime \prime} \mathrm{N} \\
30^{\circ} 49^{\prime} 18^{\prime \prime} \mathrm{E}\end{array}$ & 1312 \\
\hline \multirow[t]{5}{*}{$\begin{array}{l}\text { Phlomis grandiflora } \mathrm{H} \text {. } \\
\text { S. Thompson var. } \\
\text { grandiflora }\end{array}$} & $\begin{array}{l}\text { Antalya-Burdur } \\
\text { motoway, Dağbeli } \\
\text { province }\end{array}$ & $\begin{array}{l}37^{\circ} 38^{\prime} 44^{\prime \prime} \mathrm{N} \\
30^{\circ} 52^{\prime} 09^{\prime \prime} \mathrm{E}\end{array}$ & 855 \\
\hline & $\begin{array}{l}\text { Antalya-Isparta } \\
\text { motoway, Gökbel } \\
\text { province }\end{array}$ & $\begin{array}{l}37^{\circ} 38^{\prime} 08^{\prime \prime} \mathrm{N} \\
30^{\circ} 42^{\prime} 48^{\prime \prime} \mathrm{E}\end{array}$ & 900 \\
\hline & $\begin{array}{l}\text { Isparta- Kovada Lake } \\
\text { National Park }\end{array}$ & $\begin{array}{l}36^{\circ} 53^{\prime} 03^{\prime \prime} \mathrm{N} \\
29^{\circ} 25^{\prime} 38^{\prime \prime} \mathrm{E}\end{array}$ & 920 \\
\hline & $\begin{array}{l}\text { Burdur- Gölhisar } \\
\text { county, Ballık province }\end{array}$ & $\begin{array}{l}37^{\circ} 11^{\prime} 11^{\prime \prime} \mathrm{N} \\
30^{\circ} 11^{\prime} 02^{\prime \prime} \mathrm{E}\end{array}$ & 1427 \\
\hline & $\begin{array}{l}\text { Isparta-Sütçüler county, } \\
\text { Tota mountain } \\
\text { Bögülüyurt province }\end{array}$ & $\begin{array}{l}37^{\circ} 35^{\prime} 01^{\prime \prime} \mathrm{N} \\
31^{\circ} 04^{\prime} 34^{\prime \prime} \mathrm{E}\end{array}$ & 1580 \\
\hline $\begin{array}{l}\text { Phlomis leucophracta P. } \\
\text { H. Davis \& Hub.-Mor. }\end{array}$ & $\begin{array}{l}\text { Burdu- Bucak county, } \\
\text { Karg1 Taşdibi province }\end{array}$ & $\begin{array}{l}37^{\circ} 34^{\prime} 05^{\prime \prime} \mathrm{N} \\
30^{\circ} 50^{\prime} 43^{\prime \prime} \mathrm{E}\end{array}$ & 176 \\
\hline
\end{tabular}




\begin{tabular}{|c|c|c|c|}
\hline Taxa & Province & Location & Altitude (m) \\
\hline & $\begin{array}{l}\text { Isparta- Eğirdir county, } \\
\text { Aşağ1gökdere Akbelenli } \\
\text { province }\end{array}$ & $\begin{array}{l}37^{\circ} 38^{\prime} 16^{\prime \prime} \mathrm{N} \\
30^{\circ} 43^{\prime} 41^{\prime \prime} \mathrm{E}\end{array}$ & 420 \\
\hline & $\begin{array}{l}\text { Burdur- Ağlasun } \\
\text { county, Çamlıdere } \\
\text { village }\end{array}$ & $\begin{array}{l}37^{\circ} 15^{\prime} 30^{\prime \prime} \mathrm{N} \\
30^{\circ} 48^{\prime} 39^{\prime \prime} \mathrm{E}\end{array}$ & 690 \\
\hline \multirow[t]{3}{*}{ Phlomis lycia D. Don } & $\begin{array}{l}\text { Burdur- Bucak county, } \\
\text { Boğazköy province }\end{array}$ & $\begin{array}{l}37^{\circ} 09^{\prime} 15^{\prime \prime} \mathrm{N} \\
30^{\circ} 17^{\prime} 35^{\prime \prime} \mathrm{E}\end{array}$ & 783 \\
\hline & $\begin{array}{l}\text { Burdur- Bucak county, } \\
\text { Uğurlu province }\end{array}$ & $\begin{array}{l}37^{\circ} 09^{\prime} 22^{\prime \prime} \mathrm{N} \\
30^{\circ} 30^{\prime} 05^{\prime \prime} \mathrm{E}\end{array}$ & 823 \\
\hline & $\begin{array}{l}\text { Burdur- Bucak county, } \\
\text { Çubuk Beli province }\end{array}$ & $\begin{array}{l}37^{\circ} 14^{\prime} 23^{\prime \prime} \mathrm{N} \\
30^{\circ} 29^{\prime} 50^{\prime \prime} \mathrm{E}\end{array}$ & 908 \\
\hline \multirow[t]{7}{*}{ Phlomis nissolii L. } & Afyon- Çay county & $\begin{array}{l}37^{\circ} 47^{\prime} 08^{\prime \prime} \mathrm{N} \\
30^{\circ} 45^{\prime} 54^{\prime \prime} \mathrm{E}\end{array}$ & 830 \\
\hline & $\begin{array}{l}\text { Isparta-Sav county, } \\
\text { Yazıöögüt village }\end{array}$ & $\begin{array}{l}37^{\circ} 46^{\prime} 09^{\prime \prime} \mathrm{N} \\
30^{\circ} 37^{\prime} 13^{\prime \prime} \mathrm{E}\end{array}$ & 989 \\
\hline & $\begin{array}{l}\text { Isparta-Şarkikaraağaç } \\
\text { county, Belceğiz village }\end{array}$ & $\begin{array}{l}38^{\circ} 00^{\prime} 25^{\prime \prime} \mathrm{N} \\
31^{\circ} 17^{\prime} 32^{\prime \prime} \mathrm{E}\end{array}$ & 1203 \\
\hline & $\begin{array}{l}\text { Isparta-Yalvaç county, } \\
\text { İleği village }\end{array}$ & $\begin{array}{l}37^{\circ} 41^{\prime} 46^{\prime \prime} \mathrm{N} \\
31^{\circ} 22^{\prime} 18^{\prime \prime} \mathrm{E}\end{array}$ & 1224 \\
\hline & $\begin{array}{l}\text { Isparta- Şarkikaraağaç } \\
\text { county, Salur village }\end{array}$ & $\begin{array}{l}37^{\circ} 58^{\prime} 03^{\prime \prime} \mathrm{N} \\
31^{\circ} 17^{\prime} 45^{\prime \prime} \mathrm{E}\end{array}$ & 1236 \\
\hline & $\begin{array}{l}\text { Isparta- Aksu county, } \\
\text { Zindan cave province }\end{array}$ & $\begin{array}{l}38^{\circ} 57^{\prime} 02^{\prime \prime} \mathrm{N} \\
31^{\circ} 02^{\prime} 19^{\prime \prime} \mathrm{E}\end{array}$ & 1280 \\
\hline & $\begin{array}{l}\text { Isparta- Urban forest, } \\
\text { Sidre province }\end{array}$ & $\begin{array}{l}37^{\circ} 44^{\prime} 28^{\prime \prime} \mathrm{N} \\
30^{\circ} 33^{\prime} 18^{\prime \prime} \mathrm{E} \\
\end{array}$ & 1316 \\
\hline \multirow[t]{2}{*}{$\begin{array}{l}\text { Phlomis pungens Willd. } \\
\text { var. pungens (Silvanok) }\end{array}$} & $\begin{array}{l}\text { Isparta- Sorgun Yaylas1 } \\
\text { province }\end{array}$ & $\begin{array}{l}37^{\circ} 50^{\prime} 12^{\prime \prime} \mathrm{N} \\
31^{\circ} 06^{\prime} 15^{\prime \prime} \mathrm{E}\end{array}$ & 1488 \\
\hline & Burdur- Gölhisar county & $\begin{array}{l}37^{\circ} 44^{\prime} 28^{\prime \prime} \mathrm{N} \\
29^{\circ} 24^{\prime} 24^{\prime \prime} \mathrm{E}\end{array}$ & 1649 \\
\hline \multirow[t]{2}{*}{ Phlomis samia L. } & $\begin{array}{l}\text { Isparta- Kovada Lake } \\
\text { National Park }\end{array}$ & $\begin{array}{l}37^{\circ} 38^{\prime} 53^{\prime \prime} \mathrm{N} \\
30^{\circ} 52^{\prime} 08^{\prime \prime} \mathrm{E}\end{array}$ & 942 \\
\hline & $\begin{array}{l}\text { Isparta-Yenişarbademli } \\
\text { county Pınargözü } \\
\text { province }\end{array}$ & $\begin{array}{l}37^{\circ} 42^{\prime} 06^{\prime \prime} \mathrm{N} \\
31^{\circ} 18^{\prime} 36^{\prime \prime} \mathrm{E}\end{array}$ & 1529 \\
\hline
\end{tabular}

\section{Preparation of plant samples for GCMS analyses}

Samples belonging to three different vegetation periods, pre-bloom, bloom, and postemergence were taken from the determined areas. Collected leaves and flower samples were transported to the laboratory after placement in paper packages and without delay or exposure to sunlight. All materials were dried. 


\section{Determination of volatile compounds}

Volatile components of Phlomis leaves and flowers were determined using a solid phase microextarction method (SPME) (Vichy et al., 2003). For this aim, after drying the plant materials at room temperature $\left(25^{\circ} \mathrm{C}\right)$, two grams from each sample were bottled and heated 15 minutes in $60^{\circ} \mathrm{C}$. A proper edge injector sank to the bottom and was absorbed for 30 minutes. Compounds held to the fiber edge were injected to a GC injection block and given five minutes for desorbing. The model for the used SPME Fiber was 75UMCAR/PDMSFUSED-SILICA, Supelco, USA, PA.

Gas chromatography-mass spectrometry equipment (GC-MS, Shimadzu QP 5050, Japan) was used in order to determine volatile compounds. HP-5 MS (30 m x $0.25 \mathrm{~mm}$ length and 0.25 um film was used in device and helium was used as the column and carrier gas (10 psi flow rate). The temperature of the injection block was $240{ }^{\circ} \mathrm{C}$ and detector temperature was $250{ }^{\circ} \mathrm{C}$.

\section{Method for statistical data}

Non-parametrical tests were used, as the ratios determined for each volatile oil compound did not meet preconditions for parametric tests. The Kruskal-Wallis test, which is a non-parametrical test, was used in determining inter-species differences. The Friedman test, a non-parametrical test, was used for determining differences in different vegetation periods. The Bonferroni-Dunn method, which is a multi-compare method, was used for determining differences between the media.

\section{Results}

Leaf and flower volatile compounds of Phlomis armeniaca, P. bourgaei, $P$. grandiflora var. grandiflora, $P$. leucophracta, Phlomis lycia, P. nissolii, P. pungens var. pungens, $P$. samia were determined by SPME analysis (solid-based micro extraction method).

SPME analysis found 54 volatile components of Phlomis armeniaca, 62 of Phlomis bourgaei, 60 of Phlomis grandiflora var. grandiflora, 70 of Phlomis leucophracta, 62 of Phlomis lycia, 53 of Phlomis nissolii, 70 of Phlomis pungens var. pungens and 64 of Phlomis samia. These results were given in Table 2.

(E)-2-hexenal, $\beta$-caryophyllene and germakren-D were volatile components of Phlomis armeniaca. Volatile components during the pre-bloom period were: (E)-2hexenal (11.64\%), $\beta$-caryophyllene $(15.73 \%)$ and germakren D $(23.45 \%)$; during the bloom period (E)-2-hexenal (12.12\%), $\beta$-caryophyllene $(16.63 \%)$ and germakren-D $(27.22 \%)$ and during the post-emergence period were: (E)-2-hexenal $(10.07 \%), \beta$ caryophyllene $(11.55 \%)$ and germakren-D $(25.03 \%)$.

$\alpha$-cubebene, $\beta$-caryophyllene and germakren-D were volatile components of $P$. bourgaei. Specifically: $\alpha$-cubebene $(15.55 \%), \quad \beta$-caryophyllene $(20.80 \%)$ and germakren-D (12.41\%) ratios in the pre-bloom period, $\alpha$-cubebene $(16.04 \%), \beta$ caryophyllene $(21.98 \%)$ and germakren-D $(15.12 \%)$ in the bloom period; and $\alpha$ cubebene $(13.92 \%), \beta$-caryophyllene $(14.73 \%)$ and germakren-D $(11.21 \%)$ in the post emergence period. 
Table 2. Volatile components of leaf and flower according to different vegetation periods

\begin{tabular}{|c|c|c|c|c|c|c|c|c|c|c|c|c|c|c|c|c|c|c|c|c|c|c|c|c|}
\hline \multicolumn{25}{|c|}{ Volatile components } \\
\hline & \multicolumn{3}{|c|}{ P. armeniaca } & \multicolumn{3}{|c|}{ P. bourgaei } & \multicolumn{3}{|c|}{ P. grandiflora } & \multicolumn{3}{|c|}{ P. leucophracta } & \multicolumn{3}{|c|}{ P. lycia } & \multicolumn{3}{|c|}{ P. nissolii } & \multicolumn{3}{|c|}{ P. pungens var. pungens } & \multicolumn{3}{|c|}{ P. samia } \\
\hline Components & P.B.. & B.P. & P.E. & P.B.. & B.P. & P.E. & P.B.. & B.P. & P.E. & P.B.. & B.P. & P.E. & P.B.. & B.P. & P.E. & P.B.. & B.P. & P.E. & P.B.. & B.P. & P.E. & P.B.. & B.P. & P.E. \\
\hline Dimethyl sulfide & 0.26 & 1.23 & 1.06 & 0.3 & 0.28 & - & 0.3 & 0.13 & 0.1 & - & - & 0.26 & - & - & - & - & - & 1.89 & 0.82 & 0.18 & - & - & - & - \\
\hline 2-methyl-Propanal & - & 0.86 & 0.66 & - & - & - & - & 0.26 & - & - & - & 0.14 & - & - & - & - & - & 0.26 & 0.95 & - & - & - & - & - \\
\hline Isobuttyl alcohol & 0.3 & - & - & 0.59 & 0.43 & - & 0.6 & - & - & - & - & - & - & - & - & - & - & - & - & 0.17 & - & - & - & - \\
\hline 2-Butenal & - & - & - & - & - & - & - & - & - & 1.09 & 1 & 0.67 & 0.47 & 0.24 & 0.58 & 0.14 & 0.6 & - & - & 1.17 & 0.64 & 0.68 & - & - \\
\hline 3-Methylbutanal & 0.28 & 0.97 & 1.31 & 1.18 & 0.61 & 0.18 & 0.13 & 0.26 & 0.75 & 0.38 & 0.39 & 1.67 & 0.03 & 0.06 & 0.08 & 0.23 & 0.16 & 0.41 & 1.29 & 4.41 & 0.55 & 0.45 & 0.68 & 0.88 \\
\hline 2-Methylbutanal & 0.33 & 0.55 & 1.07 & 1.11 & 0.47 & - & 0.17 & 0.24 & 0.65 & 0.52 & 0.61 & 1.12 & 0.06 & 0.1 & 0.09 & - & - & - & 1.08 & 4.33 & 0.25 & 0.86 & - & - \\
\hline $\begin{array}{l}\text { Methyl propyl } \\
\text { ketone }\end{array}$ & - & 1 & 0.55 & - & - & - & - & - & - & - & - & - & - & - & - & 1.06 & - & - & - & - & - & - & - & - \\
\hline n-Pentenal & - & - & - & - & - & - & - & - & - & 0.54 & 0.47 & 0.56 & 0.45 & 0.15 & 0.33 & - & - & - & - & 1.16 & 0.32 & 0.7 & - & - \\
\hline $\begin{array}{c}\text { Ethyl Vinly } \\
\text { Carbinol } \\
\end{array}$ & - & - & - & 0.19 & - & - & - & - & - & - & - & - & - & - & - & - & - & - & - & 0.93 & - & - & - & - \\
\hline Ethyl Propyl Keton & - & - & - & - & - & 0.13 & - & - & - & - & - & - & - & - & - & - & - & - & - & - & - & - & - & - \\
\hline Furan, 2-ethyl- & 0.39 & 0.75 & 3.11 & - & 0.89 & 0.32 & 0.51 & 0.36 & 0.12 & 0.35 & 0.3 & 2.09 & - & 0.23 & 0.32 & 0.88 & 0.39 & 0.59 & 1.42 & 1.1 & 1.11 & 0.95 & - & - \\
\hline Ethyl vinly ketone & - & - & - & - & - & - & 0.11 & - & - & 0.34 & 0.12 & 0.66 & - & - & - & - & - & - & - & 0.52 & - & 0.62 & - & - \\
\hline $\begin{array}{l}\text { trans-3-Penten-2- } \\
\text { one }\end{array}$ & - & - & - & - & - & - & - & - & - & 0.16 & 0.18 & 0.1 & - & 0.04 & 0.17 & - & - & - & - & - & - & - & - & - \\
\hline 3-Methyl-1-butanol & - & - & - & - & - & - & - & - & - & - & - & - & - & - & - & - & - & - & 0.28 & 1.57 & - & - & - & - \\
\hline 2-Methyl-1-butanol & - & - & - & - & - & - & - & - & - & - & - & - & - & - & - & - & - & - & - & 0.55 & - & - & - & - \\
\hline (E)-2-Pentenal & - & 0.24 & 0.25 & - & - & - & - & - & - & 0.18 & 0.2 & 0.75 & 0.1 & 0.12 & 0.21 & 0.45 & 0.32 & 0.37 & 0.31 & 0.57 & 0.59 & 0.56 & - & 0.34 \\
\hline (Z)-2-Pentenol & - & - & - & - & - & - & - & - & - & - & - & - & - & - & - & - & - & - & - & 0.44 & 0.32 & 0.14 & - & - \\
\hline 3-Methyl-2-butanol & - & - & - & - & - & - & - & - & - & - & - & 0.37 & - & - & - & - & - & - & - & - & - & - & 0.47 & 0.48 \\
\hline 1-Pentanol & - & - & - & - & - & - & - & - & - & - & - & - & - & - & - & - & - & - & - & 0.77 & - & - & - & - \\
\hline n-Hexanal & 0.74 & 1.36 & 1.95 & 0.79 & 0.85 & 0 & 0.39 & 0.11 & 0.2 & 4.89 & 3.54 & 3.27 & 2.95 & 1.56 & 3.88 & 0.58 & 1.87 & 1.27 & 2.68 & 4.86 & 3.73 & 2.69 & 2.5 & 4.1 \\
\hline (E)-2-Hexenal & 11.64 & 12.12 & 10.07 & 5.52 & 3.82 & 0.5 & 4.34 & 0.51 & 1.02 & 7.5 & 8.74 & 6.1 & 3.78 & 3.92 & 6.85 & 8.57 & 10.57 & 9.05 & 16.87 & 17.6 & 12.68 & 9.04 & 2.98 & 3.66 \\
\hline Z-3-hexenol & - & 0.93 & - & - & - & - & - & - & - & 0.22 & 0.44 & 1.91 & 0.11 & 0.18 & 0.35 & 0.76 & 0.18 & - & - & 0.33 & 2.52 & 1.24 & - & - \\
\hline cis-3-Hexene-1-ol & 0.8 & - & 1.9 & 0.66 & - & - & - & - & - & - & - & - & - & - & - & - & - & - & 2.65 & 3.88 & - & - & - & - \\
\hline cis-Hex-2-en-1-ol & 0.27 & 0.2 & 1.83 & - & - & - & - & - & - & - & - & - & - & - & - & - & - & - & 0.34 & 1.3 & - & - & - & - \\
\hline
\end{tabular}

APPLIED ECOLOGY AND ENVIRONMENTAL RESEARCH 14(3): 753-772.

http://www.aloki.hu • ISSN 15891623 (Print) • ISSN 17850037 (Online)

DOI: http://dx.doi.org/10.15666/aeer/1403_753772

(c) 2016, ALÖKI Kft., Budapest, Hungary 


\begin{tabular}{|c|c|c|c|c|c|c|c|c|c|c|c|c|c|c|c|c|c|c|c|c|c|c|c|c|}
\hline 2-Hexenol, (E)- & - & - & - & - & - & - & - & - & - & - & - & - & - & - & - & 0.83 & - & - & - & - & 0.44 & - & - & - \\
\hline Hexanol <n-> & 0.34 & 0.5 & 3 & - & - & - & - & - & - & 0.16 & 0.25 & 0.51 & 0.05 & 0.17 & 0.09 & 0.81 & 0.05 & 1.17 & 1.3 & 2.17 & 2.2 & 0.3 & - & 0.42 \\
\hline $\begin{array}{l}\text { Amyl methyl } \\
\text { ketone }\end{array}$ & - & - & - & - & - & - & - & - & - & - & - & - & - & - & - & - & - & - & - & - & - & - & 0.46 & 0.36 \\
\hline n-Heptanal & - & - & - & 0.29 & 0.29 & 0.29 & - & - & - & 0.92 & 0.85 & 2.3 & 0.15 & 0.15 & 0.24 & - & - & - & - & 0.23 & 0.4 & 1.02 & - & 9.75 \\
\hline 2,4-Hexadienal & - & - & - & - & - & - & - & - & - & 0.42 & 0.56 & 0.2 & 0.26 & 0.1 & 0.43 & - & - & - & - & 0.54 & - & 0.19 & - & - \\
\hline $\begin{array}{l}\text { Sorbaldehyde, } \\
\text { (E,E) }\end{array}$ & - & - & - & - & - & - & - & - & - & - & - & 2.15 & - & - & - & - & - & - & 0.68 & - & - & - & - & 2.61 \\
\hline$\alpha$-Thujene & - & - & - & 0.31 & 0.39 & 0.43 & 1.64 & 1.43 & 1.22 & 0.54 & 0.51 & 0.5 & 0.18 & 0.39 & 0.33 & 0.68 & 0.13 & 0.54 & 0.77 & 1.48 & 0.66 & - & - & - \\
\hline .. -Pinene & 0.74 & 0.93 & 1.44 & 0.11 & 2.13 & 2.8 & 25.97 & 26.4 & 18.95 & 2.85 & 2.72 & 1.92 & 1.26 & 2.24 & 2.03 & 6.86 & 6.93 & 6.43 & 4.91 & 4.07 & 3.85 & 0.26 & 2.2 & 1.1 \\
\hline (E)-2-Heptenal & - & - & - & - & - & - & - & - & - & 1.33 & 1.26 & 1.25 & 0.67 & 0.35 & 0.79 & - & - & - & - & - & 0.23 & 0.39 & - & - \\
\hline Benzaldehyde & 2.63 & 3.8 & 0.39 & 0.49 & 0.86 & - & 0.88 & 0.3 & 0.11 & 2.5 & 1.98 & 2.77 & 0.8 & 1.99 & 1.9 & 0.58 & 0.24 & 4.03 & 5.42 & 2.88 & 3.12 & 5.36 & 2.51 & 2.08 \\
\hline \begin{tabular}{|l|} 
Methyl 2-hexenoate \\
\end{tabular} & - & - & - & - & - & - & - & - & - & - & - & - & - & - & - & - & - & 0.33 & - & - & - & - & - & - \\
\hline $\begin{array}{c}\text { 2-Hexenoic acid, } \\
\text { methyl ester }\end{array}$ & - & 1.02 & - & - & - & - & - & - & - & - & - & - & - & - & - & - & - & - & - & - & - & - & - & - \\
\hline Sabinene & - & - & - & - & - & - & - & - & - & - & - & - & - & - & - & - & - & - & - & 0.4 & 0.23 & - & - & - \\
\hline .ß.-Phellandrene & - & - & - & - & - & - & 0.44 & 0.41 & 0.39 & - & - & - & - & - & - & - & - & - & - & - & - & - & - & - \\
\hline$\beta$-Pinene & - & - & - & - & - & - & 0.79 & 0.13 & 0.66 & 0.15 & 0.22 & 0.1 & - & - & - & - & - & - & - & 0.16 & 0.33 & - & - & - \\
\hline Amyl vinyl ketone & - & - & - & - & - & - & - & - & - & 0.24 & 0.23 & 0.39 & 0.15 & 0.05 & 0.17 & - & - & - & - & - & - & - & - & - \\
\hline Vinly amly carbinol & 2.13 & 5.45 & 5.55 & 3.02 & 0.25 & 0.94 & 0.18 & 0.09 & 0.15 & 1.06 & 0.83 & 0.7 & 1.38 & 0.75 & 1.52 & 2.71 & 1.44 & 3.83 & 12.85 & 18.44 & 18.6 & 0.69 & 0.55 & 0.73 \\
\hline $\begin{array}{l}\text { 4-Pentene-1-yl } \\
\text { acetate }\end{array}$ & - & - & 0.38 & - & - & - & - & - & - & - & - & - & - & - & - & - & - & - & - & - & - & - & - & - \\
\hline \begin{tabular}{|c|} 
6-Methyl-5-hepten- \\
2-one
\end{tabular} & - & - & - & 0.21 & 0.45 & 0 & 0.38 & 0.31 & 0.17 & 0.42 & 0.44 & 1 & 0.2 & 0.24 & 0.35 & - & - & - & - & - & - & 0.42 & 0.44 & 0.52 \\
\hline Amyl ethyl ketone & 0.3 & 0.78 & 1.75 & - & - & 0.66 & - & - & - & - & - & - & - & - & - & 1.96 & - & 0.82 & 1.79 & 2.37 & - & - & - & - \\
\hline ß.-Myrcene & - & - & - & - & 1.15 & 0.87 & 0.47 & 0.98 & 0.87 & 2.15 & 2.28 & 1.76 & 0.86 & 2.62 & 1.47 & 3.18 & 0.52 & 1.46 & 0.88 & 0.82 & 1.63 & 0.27 & - & - \\
\hline $\begin{array}{l}\text { (R,S)-2-Propyl-5- } \\
\text { Oxohexanal }\end{array}$ & - & - & - & - & - & - & - & - & - & - & - & 0.57 & - & - & - & - & - & - & - & - & - & - & - & 1.26 \\
\hline Fenehone & 0.32 & - & - & 0.16 & - & - & - & - & - & - & - & - & - & - & - & - & - & - & - & - & - & - & - & - \\
\hline $\begin{array}{c}\text { 3-(2-methylpropyl) } \\
\text { Ccyclohexene }\end{array}$ & - & 0.6 & - & - & - & - & - & - & - & - & - & - & - & - & - & - & - & - & - & - & - & - & - & - \\
\hline $\begin{array}{c}4- \\
\text { Ethylcyclohexanol } \\
\end{array}$ & - & - & 1.54 & - & - & - & - & - & - & - & - & - & - & - & - & - & - & - & - & - & - & - & - & - \\
\hline
\end{tabular}




\begin{tabular}{|c|c|c|c|c|c|c|c|c|c|c|c|c|c|c|c|c|c|c|c|c|c|c|c|c|}
\hline 2-Octanone & - & - & - & - & - & - & - & - & - & - & - & - & - & - & - & - & - & - & - & - & - & - & 0.85 & - \\
\hline n-Octanal & - & - & - & 0.18 & 0.36 & - & 0.12 & - & - & 2.24 & 2.11 & 1.71 & 0.41 & 0.32 & 0.78 & 0.33 & 0.24 & 0.25 & - & - & 0.85 & 0.61 & 1.11 & 1.04 \\
\hline$\alpha$-Phellandrene & - & - & - & - & - & 0.21 & 0.14 & - & - & 0.44 & 0.64 & 0.77 & 0.06 & 0.58 & 0.18 & 0.66 & 0.23 & - & - & - & 0.18 & - & - & - \\
\hline 2,4-Heptandienal & 0.18 & 0.2 & 0.37 & 0.81 & 0.32 & 0 & 0 & - & - & 1.18 & 1.48 & 5.17 & 0.41 & 0.39 & 0.72 & 0.51 & 1.11 & 0.36 & 2.39 & 1.27 & 2.82 & 1.6 & 0 & 0.65 \\
\hline p-Dichlorobenzene & 0.56 & 0.6 & 0.57 & - & 0.92 & 0.46 & 0.26 & 0.11 & 0.18 & - & - & - & - & - & - & 1.15 & - & 0.86 & - & - & - & 0.15 & 0.86 & 1.01 \\
\hline .a.-Terpinene & - & - & - & - & 0.49 & 0.38 & - & - & - & 0.68 & 1 & 0.4 & 0.08 & 0.56 & 0.21 & 0.41 & 0.15 & - & - & - & - & - & - & - \\
\hline p-Cymene & - & - & - & - & 0.31 & 0.3 & 0.42 & 0.34 & 0.4 & 1.03 & 1.29 & 1.05 & 0.33 & 0.55 & 0.6 & 1.88 & 0.32 & 1 & 0.3 & 0.53 & 1.51 & - & - & - \\
\hline Limonene & 1.53 & 1.4 & 0.69 & 0.13 & 5.84 & 4.6 & 3.36 & 2.62 & 2.29 & 13.64 & 14.56 & 10.93 & 13.7 & 17.68 & 10.65 & 20.65 & 23.75 & 16.17 & 1.58 & 0.67 & 6.83 & 1.17 & 2.16 & 2.03 \\
\hline (E)-3-Octen-2-one & - & - & - & - & - & - & - & - & - & 0.75 & 0.61 & - & 0.71 & 0.19 & 0.72 & - & - & - & - & - & - & - & - & - \\
\hline cis-Ocimene & - & - & - & - & - & - & 1.47 & 0.82 & 2.03 & - & - & - & - & - & - & - & - & - & - & - & - & - & - & - \\
\hline $\begin{array}{l}\text { Benzeneacetaldehy } \\
\text { de }\end{array}$ & 0.58 & 0.69 & - & 0.41 & 0.6 & - & 0.26 & - & - & - & - & - & - & - & - & - & - & 0.32 & 0.78 & 0.46 & - & - & - & - \\
\hline Phenylacetaldehyde & - & - & - & - & - & - & - & - & - & 0.63 & 0.71 & - & 0.21 & 0.1 & 0.63 & - & - & - & - & - & 0.51 & 0.76 & - & - \\
\hline$\beta$. Ocimene & - & - & - & - & - & 0.1 & 0.18 & 0.15 & 0.14 & 0.24 & 0.39 & - & 0.05 & 0.39 & 0.11 & 0.42 & 0.04 & - & - & - & - & - & - & - \\
\hline 2 Octenal & - & - & - & - & - & - & - & - & - & 0.93 & 0.98 & 0.63 & 0.45 & 0.46 & 0.58 & - & - & - & - & - & 0.41 & 0.2 & 1.14 & 0.88 \\
\hline 3,5-Octadien-2-one & - & 0.21 & - & - & - & - & - & - & - & 0.36 & 0.34 & - & 0.26 & 0.1 & 0.48 & - & - & - & - & - & 0.49 & 0.55 & - & - \\
\hline$\gamma$-Terpinene & - & - & - & - & 0.63 & 0.51 & - & - & - & - & - & - & - & - & - & - & - & - & - & - & - & - & - & - \\
\hline Octanol & - & - & - & - & - & - & - & - & - & 0.1 & 0.19 & - & - & - & - & - & - & - & - & - & - & - & - & - \\
\hline .a.-Terpinolene & - & - & - & - & - & - & - & - & - & 1.55 & 1.29 & 0.5 & 0.14 & 0.89 & 0.36 & 1.1 & 0.42 & 0.32 & - & - & - & - & - & - \\
\hline $\begin{array}{c}\text { Dimethylstyrene } \\
<\alpha \text {-para- }>\end{array}$ & - & - & - & - & - & - & - & - & - & 0.1 & 0.21 & - & - & - & - & - & - & - & - & - & - & - & - & - \\
\hline 2-Nonanone & - & - & - & - & 0.78 & 3.72 & - & - & - & - & - & - & - & - & - & - & - & - & - & - & - & 0.22 & 0.93 & 0.44 \\
\hline Methyl benzoate & - & - & - & - & - & - & - & - & - & 0.26 & 0.27 & - & 0.16 & 0.07 & 0.28 & - & - & - & - & - & - & - & - & - \\
\hline Linalool & 0.58 & 2.02 & - & - & - & - & - & - & 0.23 & - & - & 1.04 & - & - & 0.34 & - & - & - & 1.17 & - & - & 0.7 & 1.55 & 0.44 \\
\hline n-Nonanal & 0.54 & 0.76 & 0.42 & 1.02 & 0.73 & 0.3 & 0.3 & 0.15 & 0.6 & 3.44 & 2.61 & 2.32 & 0.7 & 1.03 & 1.24 & 1.26 & 1.41 & 0.99 & 1 & 0.39 & 7.59 & 3.5 & 3.28 & 3.17 \\
\hline 2-Hendecanone & - & - & - & 0.19 & 3.27 & 29.87 & - & - & - & - & - & - & - & - & - & - & - & - & - & - & - & - & - & - \\
\hline Phenethyl alcohol & 0.54 & - & - & - & - & - & - & - & - & - & - & - & - & - & - & - & - & - & 0.52 & 0.32 & - & 0.25 & - & - \\
\hline $\begin{array}{l}\text { a.-Campholene } \\
\text { aldehyde }\end{array}$ & - & - & - & - & - & - & - & 0.12 & 0.09 & - & - & - & - & - & - & - & - & - & - & - & - & - & - & - \\
\hline trans-Alloocimene & - & - & - & - & - & - & - & 0.08 & 0.2 & - & - & - & - & - & - & - & - & - & - & - & - & - & - & - \\
\hline D-Carvone & - & - & - & - & - & - & - & - & 0.11 & - & - & - & - & - & - & - & - & - & - & - & - & - & - & - \\
\hline Trans-2-Nonenal & - & - & - & - & - & - & - & - & - & 0.3 & 0.5 & 0.59 & - & - & - & - & - & - & - & - & - & 0.25 & 0.92 & 0.62 \\
\hline
\end{tabular}

APPLIED ECOLOGY AND ENVIRONMENTAL RESEARCH 14(3): 753-772.

http://www.aloki.hu • ISSN 15891623 (Print) • ISSN 17850037 (Online)

DOI: http://dx.doi.org/10.15666/aeer/1403_753772

(c) 2016, ALÖKI Kft., Budapest, Hungary 


\begin{tabular}{|c|c|c|c|c|c|c|c|c|c|c|c|c|c|c|c|c|c|c|c|c|c|c|c|c|}
\hline Pinocarvone & - & - & - & - & - & - & - & 0.09 & 0.09 & - & - & - & - & - & - & - & - & - & - & - & - & - & - & - \\
\hline Methyl salicylate & 1.17 & 2.15 & 0.34 & - & - & - & - & - & - & - & - & 0.6 & - & - & - & - & - & 1.29 & 1.36 & - & - & - & - & - \\
\hline (E)-2-Nonenal & - & - & - & - & - & - & - & - & - & - & - & 0 & 0.23 & 0.17 & 0.23 & - & - & - & - & - & - & - & - & - \\
\hline n-Decanal & - & - & - & - & - & - & - & - & - & 0.62 & 0.56 & 0.3 & 0.52 & 1.05 & 0.93 & 0.17 & 0.19 & - & - & - & 0.4 & 0.47 & 0.96 & 0.43 \\
\hline (E)-2-Decenal & - & - & - & - & - & - & - & - & - & 0.24 & 0.46 & 0.15 & 0.09 & - & 0.11 & - & - & - & - & - & - & - & - & - \\
\hline Hendecanal & - & - & - & - & - & - & - & - & - & 0.13 & 0.24 & - & 0.02 & - & - & - & - & - & - & - & - & - & - & - \\
\hline $\begin{array}{l}\text { Nonyl methyl } \\
\text { ketone }\end{array}$ & 0.23 & - & - & - & - & - & - & - & - & - & - & - & - & - & - & - & - & - & - & - & - & - & - & - \\
\hline 2-Hendecanone & - & - & - & - & - & - & - & - & - & - & - & - & - & - & - & - & - & - & - & - & - & - & 0.85 & - \\
\hline .a.-Cubebene & 1.11 & 1.31 & 1.9 & 15.55 & 16.04 & 13.92 & - & - & - & 3.55 & 3.3 & $2.50-$ & 1.86 & 0.29 & 2.75 & 0.27 & 0.45 & 0.27 & 0.38 & 0.41 & 0.3 & 0.42 & 1.29 & 0.92 \\
\hline Ylangene & 0.18 & - & - & - & 0.22 & 0.23 & - & - & - & - & - & - & - & - & - & - & - & - & - & - & - & - & - & - \\
\hline ...-Ylangene & - & - & - & - & - & - & - & - & - & - & - & - & - & - & - & - & - & 0.59 & - & - & - & - & 0.75 & - \\
\hline .. -Copaene & 0.8 & 1.11 & 1.22 & 3.97 & 3.1 & 3.2 & 0.18 & 0.16 & 0.54 & 0.96 & 0.79 & 2.78 & 0.93 & 1.53 & 1.21 & 1.84 & 1.98 & 0.84 & 0.5 & 0.23 & 0.6 & 9.59 & 10.59 & 9.71 \\
\hline$\beta$. Bourbonene & 0.58 & 1.15 & 2.48 & 0.31 & 0.52 & 1.81 & 0.34 & 1.05 & 1.06 & 0.79 & 0.17 & 0.92 & 0.25 & 1.12 & 0.24 & 3.86 & 1.32 & 5.85 & 0.75 & 0.41 & 3.57 & 3.43 & 7.47 & 7.13 \\
\hline$\beta$-Cubebene & - & 0.22 & - & 6.55 & 4.59 & - & - & 0.11 & - & 0.89 & 0.2 & - & 1.07 & 0.6 & 1.26 & 0.52 & 0.99 & 0.88 & - & - & 0.54 & 0.7 & - & - \\
\hline (-)-.ß.-Elemene & 0.66 & 0.66 & 0.52 & - & - & - & - & - & - & 0.19 & 0.14 & - & 0.23 & 0.67 & 0.15 & - & - & - & 0.66 & - & 0.34 & 0.6 & 0.87 & 0.44 \\
\hline $\begin{array}{c}\text { Sesquithujene <7- } \\
\text { epi-> }\end{array}$ & - & - & - & - & 1.67 & - & 0.19 & 0.2 & 0.18 & 0 & 0 & - & 0.34 & 0 & 0.5 & - & - & - & - & - & - & - & - & - \\
\hline $\mathrm{n}$-Tetradecane & - & - & - & - & - & - & - & - & - & - & - & - & - & - & - & - & - & - & - & - & - & - & - & 0.36 \\
\hline$\alpha$-Gurjunene & - & - & - & 2.1 & 1.07 & 1.05 & - & - & - & - & - & - & - & - & - & - & - & - & - & - & - & 0.84 & - & - \\
\hline .a.-Cedrene & - & - & - & 0.55 & - & - & 25.92 & 28.15 & 19.14 & - & - & - & 0.4 & 0.19 & 0.47 & - & - & - & - & - & - & - & - & - \\
\hline$\beta$-Caryophyllene & 15.73 & 16.63 & 11.55 & 20.8 & 21.98 & 14.73 & 7.78 & 6.4 & 3.91 & 22.32 & 22.45 & 20.12 & 17.63 & 23.66 & 10.63 & 11.28 & 12.5 & 10.37 & 0.74 & 1.4 & 0.65 & 13.79 & 15.2 & 13.75 \\
\hline$\beta$-Cedrene & - & - & - & - & - & - & 0.25 & - & - & 0.23 & - & - & 0.3 & 0.49 & 0.2 & - & - & - & - & - & 0.65 & 0.45 & - & - \\
\hline .. -Elemene & 4.17 & - & - & 1.67 & - & - & - & - & - & - & - & - & - & - & - & - & - & - & 4.37 & 2.36 & 2.2 & - & - & - \\
\hline 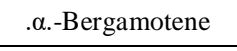 & - & - & - & - & - & - & 0.94 & 1.43 & 2.03 & - & - & - & 1.56 & 0.25 & 1.76 & - & - & - & - & - & 0.2 & 0.2 & - & - \\
\hline . $\alpha$-Cedrol & - & - & - & - & - & - & - & 0.52 & 0.45 & - & - & - & - & - & - & - & - & - & - & - & - & - & - & - \\
\hline .ঠ.-Guaiene & - & - & - & 0.61 & - & - & - & - & - & - & - & - & - & - & - & - & - & - & - & - & - & - & - & - \\
\hline . $\alpha$-Amorphene & - & - & - & - & - & - & - & - & - & - & - & - & - & - & - & - & - & 0.47 & - & - & - & - & 0.85 & - \\
\hline Alloaromadendrene & - & - & - & 4.63 & - & - & 0.17 & - & - & 1.17 & 0.56 & 0.77 & - & 0.43 & 0.41 & 1.64 & 0.29 & - & - & - & 0.29 & - & - & - \\
\hline (E)- $\beta$-Farnesene & 14.87 & 0.21 & 6.87 & - & - & - & 1.8 & - & 1.28 & 3.57 & 2.96 & 2.4 & 4.76 & 3.71 & 10.23 & 3.86 & 4.05 & 5.86 & 11.32 & 0.43 & 1.94 & - & - & - \\
\hline Isoledene & - & - & - & - & 0.22 & - & - & - & - & - & - & - & - & - & - & - & - & - & - & - & - & - & - & - \\
\hline .a.-Humulene & - & 2.08 & - & 1.39 & 3.05 & 1.35 & - & - & - & - & - & 0.4 & - & - & - & - & - & - & - & - & - & 2.15 & - & 1.66 \\
\hline
\end{tabular}

APPLIED ECOLOGY AND ENVIRONMENTAL RESEARCH 14(3): 753-772.

http://www.aloki.hu • ISSN 15891623 (Print) • ISSN 17850037 (Online)

DOI: http://dx.doi.org/10.15666/aeer/1403_753772

○ 2016, ALÖKI Kft., Budapest, Hungary 


\begin{tabular}{|c|c|c|c|c|c|c|c|c|c|c|c|c|c|c|c|c|c|c|c|c|c|c|c|c|}
\hline Cyclosativene & - & - & - & 5.23 & - & - & 0.64 & - & 0.68 & - & - & - & - & - & - & 2.66 & - & - & - & - & - & - & - & - \\
\hline .3.-Humulene & - & - & - & - & - & - & - & - & 7.52 & - & - & - & - & - & - & - & - & - & - & - & - & - & - & - \\
\hline Farnesol & - & - & - & - & - & - & - & 1.19 & 1.1 & - & - & - & - & - & - & - & - & - & - & - & - & - & - & - \\
\hline $\begin{array}{l}\text { Cadina-1(6),4- } \\
\text { diene }<10 \beta \mathrm{H}->\end{array}$ & 0.51 & 0.71 & 0.37 & 0.55 & 0.63 & 1.07 & - & - & - & 0.4 & 0.21 & 0.1 & 0.46 & 0.78 & 0.44 & - & 1.62 & 0.47 & - & - & 0.63 & 0.77 & 0.63 & 0.59 \\
\hline . $\beta$.-Selinene & - & - & - & - & - & 0.15 & - & - & - & - & - & - & - & - & - & - & - & - & - & - & - & - & - & - \\
\hline $\begin{array}{c}\text { Epi- } \\
\text { Bicyclosesquiphella } \\
\text { ndrene }\end{array}$ & 1.09 & 0.75 & 2.09 & 0.26 & 0.46 & 0.67 & - & - & - & - & - & 0.39 & - & - & - & 0.26 & - & 0.74 & 0.29 & - & - & - & 1.74 & 1.66 \\
\hline Germacrene-D & 23.45 & 27.22 & 25.03 & 12.41 & 15.12 & 11.21 & 4 & 5.17 & 5.4 & 6.46 & 8.32 & 7.47 & 15.66 & 21.88 & 11.76 & 12.27 & 20.73 & 10.44 & 7.78 & 9.84 & 8.25 & 21.01 & 23.44 & 18.9 \\
\hline Ionone & - & - & - & - & - & - & 0.16 & - & 1.28 & - & - & - & - & - & - & - & - & - & - & - & - & - & - & - \\
\hline$\alpha$-Curcumene & - & - & - & - & - & - & 11.96 & 13.92 & 13.24 & - & - & - & - & - & - & - & - & - & - & - & - & - & - & - \\
\hline Bicyclogermacrene & 0.63 & 1.34 & 2.5 & - & - & - & - & - & - & - & - & - & - & - & - & - & - & - & 1.14 & 1.22 & - & 0.6 & - & - \\
\hline Zingiberene & - & - & - & - & 0.41 & - & - & - & - & - & - & - & 6.06 & 0.33 & 5.17 & - & - & - & - & - & - & - & - & - \\
\hline Valencene & - & - & - & 1.59 & - & - & - & - & - & - & - & - & - & - & - & - & - & - & - & - & - & - & - & - \\
\hline$\alpha$ Bisabolol & - & - & - & - & - & 0.81 & - & - & - & - & - & - & - & - & - & - & - & - & - & - & - & - & - & - \\
\hline$\alpha$-Muurolene & 0.53 & 0.61 & 0.8 & 0.3 & 0.37 & 0.37 & - & - & - & - & - & - & - & - & - & 0.19 & 0.91 & 0.27 & - & - & - & 0.59 & 1.5 & 0.58 \\
\hline$\beta$-Muurolene & - & - & - & - & - & - & - & - & - & - & - & - & 0.25 & 0.54 & 0.2 & - & - & - & - & - & - & - & - & - \\
\hline Eremophilene & - & - & - & - & - & - & - & - & 3.04 & - & - & - & - & - & - & - & - & - & - & - & - & - & - & - \\
\hline Cedr-8-e & - & - & - & - & - & - & 0.26 & 2 & 3.02 & - & - & - & - & - & - & - & - & - & - & - & - & - & - & - \\
\hline Caran-cis-3-ol & - & - & - & - & - & - & - & - & - & - & - & - & - & - & - & - & - & - & - & - & - & - & 0.61 & - \\
\hline$\beta$-Bisabolene & - & - & - & - & 0.51 & - & - & - & - & - & 0.22 & - & - & - & - & - & - & - & - & - & - & - & - & - \\
\hline a.-selinene & - & - & - & - & - & - & - & - & 1.68 & - & - & - & - & - & - & - & - & 1.87 & - & - & - & - & - & - \\
\hline$\beta$-Bisabolene & - & - & - & - & - & - & - & - & - & - & - & - & 4.96 & - & 4.33 & - & - & - & - & - & - & - & 0.66 & - \\
\hline.$\gamma$-Cadinene & 2.06 & 2 & 1.49 & 0.33 & 1.55 & 0.55 & - & - & - & 0.38 & 0.24 & 0.2 & 6.8 & 1.09 & 3.71 & 0.63 & 1.8 & 0.4 & 0.36 & - & 0.61 & 0.82 & 1.71 & 0.91 \\
\hline$\delta$-Cadinene & 1.39 & 1.44 & 2.46 & 1.31 & 1.07 & 1.27 & - & 0.08 & 0.1 & 0.71 & 0.82 & 1.18 & 0.83 & 1.35 & 0.62 & 0.64 & 1.26 & 0.61 & 0.82 & - & 1.02 & 6.6 & 5.29 & 3.55 \\
\hline $\begin{array}{c}\beta- \\
\text { Sesquiphellandrene }\end{array}$ & - & - & - & - & - & - & 0.21 & 0.21 & 0.15 & 0.17 & 0.47 & - & 1.38 & 0.37 & 1.08 & - & - & - & - & - & - & - & - & - \\
\hline Germacrene B & 4.48 & 1.24 & - & 2.26 & - & - & - & - & - & - & - & - & - & - & - & 0.55 & 0.22 & 4.69 & 4.11 & - & 1.28 & - & - & - \\
\hline (+)-spathulenol & 0.17 & - & - & - & - & - & - & - & 0.37 & - & - & - & - & - & - & - & - & 0.39 & - & - & - & - & - & - \\
\hline $\begin{array}{c}\text { Isolongifolene, } \\
4,5,9,10 \text {-dehydro- }\end{array}$ & - & - & - & - & - & - & - & 0.18 & - & - & - & - & - & - & - & - & - & - & - & - & - & - & - & - \\
\hline
\end{tabular}

APPLIED ECOLOGY AND ENVIRONMENTAL RESEARCH 14(3): 753-772.

http://www.aloki.hu • ISSN 15891623 (Print) • ISSN 17850037 (Online)

DOI: http://dx.doi.org/10.15666/aeer/1403_753772

๔ 2016, ALÖKI Kft., Budapest, Hungary 


\begin{tabular}{|c|c|c|c|c|c|c|c|c|c|c|c|c|c|c|c|c|c|c|c|c|c|c|c|c|}
\hline $\begin{array}{l}\text { (-)-Caryophyllene } \\
\text { oxide }\end{array}$ & 0.21 & - & - & - & - & 0.2 & 0.79 & 1.22 & 1.19 & - & - & - & 0.71 & 0.16 & 0.43 & 0.37 & 0.62 & 0.98 & - & - & - & - & - & 0.5 \\
\hline (2-Ethylhexyl)Ether & - & - & - & - & - & - & - & - & - & - & - & 1.32 & - & - & - & - & - & - & - & - & - & - & - & 0.34 \\
\hline $\begin{array}{c}\text { p-Menthane, 2,3- } \\
\text { dibromo-8-phenyl- }\end{array}$ & - & - & - & - & - & - & 0.29 & 1.21 & 0.7 & - & - & - & - & - & - & - & - & - & - & - & - & - & - & - \\
\hline$\alpha$-Elemol & - & - & - & - & - & - & 0.21 & - & - & - & - & - & - & - & - & - & - & - & - & - & - & - & - & - \\
\hline Ar-tumerone & - & - & - & - & - & - & - & 0.11 & 0 & - & - & - & - & - & - & - & - & - & - & - & - & - & - & - \\
\hline $\begin{array}{l}\text { Androstan-17-one. } \\
\text { 3-ethyl-3-hydroxy- } \\
\text { (5.alpha.)- }\end{array}$ & - & - & - & - & - & - & - & 0.29 & 0.22 & - & - & - & - & - & - & - & - & - & - & - & - & - & - & - \\
\hline Nonadecane & - & - & - & - & - & - & - & - & - & 0.44 & 0.59 & - & 1.12 & 0.38 & 1.12 & - & - & - & - & - & 0.71 & 0.18 & - & - \\
\hline
\end{tabular}


Volatile components of Phlomis grandiflora var. grandiflora were: $\alpha$-pinene (25.97\%), $\alpha$-cedrene $(25.92 \%)$ and $\alpha$-curcumene $(11.96 \%)$ in the pre-bloom period; $\alpha$ pinene $(26.40 \%), \alpha$-cedrene $(28.15 \%)$ and $\alpha$-curcumene $(13.92 \%)$ in the bloom period; and $\alpha$-pinene $(18.95 \%), \alpha$-cedrene $(19.14 \%)$ and $\alpha$-curcumene $(13.24 \%)$ in the postemergence period.

(E)-2-hexenal, limonene and $\beta$-caryophyllene were volatile components of $P$. leucophracta. Specifically in the pre-bloom period: (E)-2-hexenal (7.50\%), limonene $(13.64 \%)$ and $\beta$-caryophyllene (22.32\%); in the bloom period (E)-2-hexenal (8.74\%), limonene $(14.56 \%)$ and $\beta$-caryophyllene $(22.45 \%)$ and in the post emergence period (E)-2-hexenal (6.10\%), limonene (10.93\%) and $\beta$-caryophyllene (20.12\%) were determined.

Limonene, $\beta$-caryophyllene and germacrene-D were volatile components of Phlomis lycia. Specifically limonene $(13.70 \%), \beta$-caryophyllene $(17.63 \%)$ and germacrene-D $(15.66 \%)$ in pre-bloom period; limonene $(17.68 \%), \beta$-caryophyllene $(23.66 \%)$ and germacrene-D $(21.88 \%)$ in the bloom period and also limonene $(10.65 \%), \beta$ caryophyllene (10.63\%) and germacrene-D (11.76\%) in the post-emergence period.

For Phlomis nissolii L., the volatile components were: limonene, $\beta$-caryophyllene and germacrene-D. Specifically, limonene (20.65\%), $\beta$-caryophyllene (11.28\%) and germacrene-D (12.27\%) in the pre-bloom period; limonene $(23.75 \%), \beta$-caryophyllene $(12.50 \%)$ and germacrene D (20.73\%) in the bloom period; and limonene (16.17\%), $\beta$ caryophyllene $(10.37 \%)$ and germacrene D (10.44\%) in the post-emergence period.

(E)-2-hexenal, vinly amyl carbinol and germacrene-D were volatile components of Phlomis pungens var. pungens. In the pre-bloom period, (E)-2-hexenal (16.87\%), vinly amyl carbinol (12.85\%) and germacrene-D (7.78\%); in the bloom period (E)-2-hexenal (17.60\%), vinly amyl carbinol $(18.44 \%)$ and germacrene-D $(9.84 \%)$ and in the postemergence period (E)-2-hexenal (12.68\%), vinly amyl carbinol (18.60\%) and germacrene-D $(8.25 \%)$.

The volatile components of $P$. samia were $\alpha$-copaene, $\beta$-caryophyllene and germacrene-D. Specifically $\alpha$-copaene (9.59\%), $\beta$-caryophyllene (13.79\%) and germacrene-D (21.01\%) in the pre bloom period; $\alpha$-copaene $(10.59 \%), \beta$-caryophyllene $(15.20 \%)$ and germacrene-D $(23.44 \%)$ in the bloom period and $\alpha$-copaene $(9.71 \%), \beta$ caryophyllene $(13.75 \%)$ and germacrene-D $(18.90 \%)$ in the post-emergence period.

The differences between the medians of Phlomis species were statistically important as found by the Kruskal-Wallis test in respect to volatile components per rates for (E)-2hexenal, $\alpha$-pinene, vinly amly carbinol, limonene, $\alpha$-cubebene, $\alpha$-copaene, $\alpha$-cedrene, $\beta$ caryophyllene, germacrene-D and $\alpha$-curcumene (Table 3 ).

While there was no statistically important difference between $P$. pungens and $P$. armeniaca in respect to the ordinal method for an (E)-2-hexenal compound per the multi-comparison Bonferroni-Dunn test that was applied to determine the differences between the medians, there was a statistically significant difference between $P$. pungens and $P$. bourgaei, $P$. grandiflora, $P$. leucophracta, $P$. lycia, $P$. nissolii and $P$. samia. There was no statistically significant difference between $P$. bourgaei and $P$. grandiflora. There was no statistically significant difference between these two species and $P$. lycia and $P$. samia. There was a statistically significant difference between $P$. bourgaei and $P$. grandiflora and $P$. armeniaca, P. leucophracta, $P$. nissolii and $P$. pungens.

There was a statistically significant difference between $P$. grandiflora and $P$. armeniaca, $P$. bourgaei, P. leucophracta, P. lycia, P. pungens and P. samia, however, 
there was no statistically significant difference for ratios of $\alpha$--pinene compounds according to the ordinal method between $P$. grandiflora and $P$. nissolii. While there was no statistically significant different between $P$. armeniaca and $P$. samia and $P$. bourgae $i$ and $P$. lycia. There was a statistically significant difference between $P$. armeniaca and $P$. samia and $P$. grandiflora, $P$. leucophracta, $P$. nissolii and $P$. pungens.

Table 3. Kruskal-Wallis analyses results acccording to Phlomis taxa

\begin{tabular}{|c|c|c|c|c|c|c|c|c|c|}
\hline Component & Taxa & $\mathbf{N}$ & $\mathbf{N}^{*}$ & Mean & SEMean & StDev & Median & $\mathbf{P}$ & Rank \\
\hline \multirow{8}{*}{ 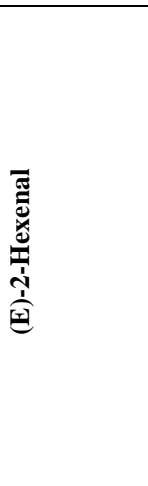 } & P. armeniaca & 3 & 0 & 11.277 & 0.619 & 1.072 & 11.640 & \multirow{8}{*}{0.009} & $19.7 \mathrm{ab}$ \\
\hline & P. bourgaei & 3 & 0 & 3.28 & 1.47 & 2.55 & 3.82 & & $6.0 \mathrm{e}$ \\
\hline & $\begin{array}{l}P . \quad \text { grandiflora } \\
\text { var. grandiflora }\end{array}$ & 3 & 0 & 1.96 & 1.20 & 2.08 & 1.02 & & $4.7 \mathrm{e}$ \\
\hline & P. leucophracta & 3 & 0 & 7.447 & 0.763 & 1.321 & 7.500 & & $13.0 \mathrm{~cd}$ \\
\hline & P. lycia & 3 & 0 & 4.85 & 1.00 & 1.73 & 3.92 & & $8.7 \mathrm{de}$ \\
\hline & P. nissolii & 3 & 0 & 9.397 & 0.603 & 1.044 & 9.050 & & $16.7 \mathrm{bc}$ \\
\hline & $\begin{array}{l}\text { P. pungens var. } \\
\text { pungens }\end{array}$ & 3 & 0 & 15.72 & 1.53 & 2.66 & 16.87 & & $23.0 \mathrm{a}$ \\
\hline & P. samia & 3 & 0 & 5.23 & 1.92 & 3.32 & 3.66 & & $8.3 \mathrm{de}$ \\
\hline Component & Taxa & $\mathbf{N}$ & $\mathbf{N}^{*}$ & Mean & SEMean & StDev & Median & $\mathbf{P}$ & Rank \\
\hline \multirow{8}{*}{ 节 } & P. armeniaca & 3 & 0 & 1.037 & 0.209 & 0.362 & 0.930 & \multirow[t]{8}{*}{0.008} & $4.7 \mathrm{e}$ \\
\hline & P. bourgaei & 3 & 0 & 1.680 & 0.808 & 1.400 & 2.130 & & $8.3 \mathrm{de}$ \\
\hline & $\begin{array}{l}P . \quad \text { grandiflora } \\
\text { var. grandiflora }\end{array}$ & 3 & 0 & 23.77 & 2.41 & 4.18 & 25.97 & & $23.0 \mathrm{a}$ \\
\hline & P. leucophracta & 3 & 0 & 2.497 & 0.291 & 0.504 & 2.720 & & $12.0 \mathrm{~cd}$ \\
\hline & P. lycia & 3 & 0 & 1.843 & 0.298 & 0.516 & 2.030 & & 9.0de \\
\hline & P. nissolii & 3 & 0 & 6.740 & 0.156 & 0.271 & 6.860 & & $20.0 \mathrm{ab}$ \\
\hline & $\begin{array}{l}P . \text { pungens var. } \\
\text { pungens }\end{array}$ & 3 & 0 & 4.277 & 0.323 & 0.559 & 4.070 & & $17.0 \mathrm{bc}$ \\
\hline & P. samia & 3 & 0 & 1.187 & 0.562 & 0.973 & 1.100 & & $6.0 \mathrm{e}$ \\
\hline Component & Taxa & $\mathbf{N}$ & $\mathbf{N}^{*}$ & Mean & SEMean & StDev & Median & $\mathbf{P}$ & Rank \\
\hline \multirow{8}{*}{ 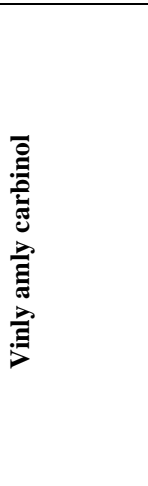 } & P. armeniaca & 3 & 0 & 4.38 & 1.12 & 1.95 & 5.45 & \multirow[t]{8}{*}{0.006} & $19.0 \mathrm{ab}$ \\
\hline & P. bourgaei & 3 & 0 & 1.403 & 0.833 & 1.442 & 0.940 & & $11.0 \mathrm{de}$ \\
\hline & $\begin{array}{l}P . \quad \text { grandiflora } \\
\text { var. } \\
\text { grandiflora }\end{array}$ & 3 & 0 & 0.1400 & 0.0265 & 0.0458 & 0.1500 & & $2.0 \mathrm{f}$ \\
\hline & P. leucophracta & 3 & 0 & 0.863 & 0.105 & 0.182 & 0.830 & & $9.7 \mathrm{de}$ \\
\hline & P. lycia & 3 & 0 & 1.217 & 0.237 & 0.410 & 1.380 & & $12.3 \mathrm{~cd}$ \\
\hline & P. nissolii & 3 & 0 & 2.660 & 0.690 & 1.196 & 2.710 & & $16.7 \mathrm{bc}$ \\
\hline & $\begin{array}{l}P . \text { pungens var. } \\
\text { pungens }\end{array}$ & 3 & 0 & 16.63 & 1.89 & 3.27 & 18.44 & & $23.0 \mathrm{a}$ \\
\hline & P. samia & 3 & 0 & 0.6567 & 0.0546 & 0.0945 & 0.6900 & & $6.3 \mathrm{ef}$ \\
\hline Component & Taxa & $\mathbf{N}$ & $\mathbf{N}^{*}$ & Mean & SEMean & StDev & Median & $\mathbf{P}$ & Rank \\
\hline \multirow{5}{*}{ 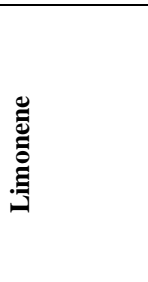 } & P. armeniaca & 3 & 0 & 1.207 & 0.261 & 0.452 & 1.400 & \multirow{5}{*}{0.011} & $4.7 \mathrm{~b}$ \\
\hline & P. bourgaei & 3 & 0 & 3.52 & 1.73 & 3.00 & 4.60 & & $9.3 b$ \\
\hline & $\begin{array}{l}\text { P. } \text { grandiflora } \\
\text { var. grandiflora }\end{array}$ & 3 & 0 & 2.757 & 0.316 & 0.548 & 2.620 & & $11.0 \mathrm{~b}$ \\
\hline & P. leucophracta & 3 & 0 & 13.04 & 1.09 & 1.89 & 13.64 & & $18.3 \mathrm{a}$ \\
\hline & P. lycia & 3 & 0 & 14.01 & 2.04 & 3.53 & 13.70 & & $19.0 \mathrm{a}$ \\
\hline
\end{tabular}




\begin{tabular}{|c|c|c|c|c|c|c|c|c|c|}
\hline & P. nissolii & 3 & 0 & 20.19 & 2.20 & 3.81 & 20.65 & & $22.7 \mathrm{a}$ \\
\hline & $\begin{array}{l}P . \text { pungens var. } \\
\text { pungens }\end{array}$ & 3 & 0 & 3.03 & 1.92 & 3.33 & 1.58 & & $8.0 \mathrm{~b}$ \\
\hline & P. samia & 3 & 0 & 1.787 & 0.311 & 0.538 & 2.030 & & $7.0 \mathrm{~b}$ \\
\hline Component & Taxa & $\mathbf{N}$ & $\mathbf{N}^{*}$ & Mean & SEMean & StDev & Median & $\mathbf{P}$ & Rank \\
\hline \multirow{8}{*}{ 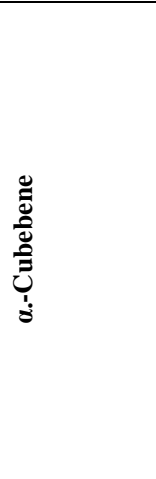 } & P. armeniaca & 3 & 0 & 1.440 & 0.237 & 0.411 & 1.310 & \multirow[t]{8}{*}{0.008} & $15.0 \mathrm{bc}$ \\
\hline & P. bourgaei & 3 & 0 & 15.170 & 0.641 & 1.110 & 15.550 & & $22.0 \mathrm{a}$ \\
\hline & $\begin{array}{l}P . \quad \text { grandiflora } \\
\text { var. grandiflora }\end{array}$ & 3 & 0 & 0.000000 & 0.000000 & 0.000000 & 0.000000 & & $2.0 \mathrm{f}$ \\
\hline & P. leucophracta & 3 & 0 & 3.425 & 0.125 & 0.177 & 3.425 & & $19.5 \mathrm{ab}$ \\
\hline & P. lycia & 3 & 0 & 1.633 & 0.719 & 1.246 & 1.860 & & $13.3 \mathrm{~cd}$ \\
\hline & P. nissolii & 3 & 0 & 0.3300 & 0.0600 & 0.1039 & 0.2700 & & $6.7 \mathrm{ef}$ \\
\hline & $\begin{array}{l}\text { P. pungens var. } \\
\text { pungens }\end{array}$ & 3 & 0 & 0.3633 & 0.0328 & 0.0569 & 0.3800 & & 8.0de \\
\hline & P. samia & 3 & 0 & 0.877 & 0.252 & 0.437 & 0.920 & & $12.0 \mathrm{cde}$ \\
\hline Component & Taxa & $\mathbf{N}$ & $\mathbf{N}^{*}$ & Mean & SEMean & StDev & Median & $\mathbf{P}$ & Rank \\
\hline \multirow{8}{*}{ 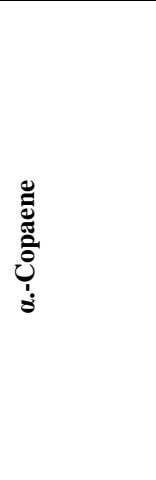 } & P. armeniaca & 3 & 0 & 1.043 & 0.126 & 0.218 & 1.110 & \multirow[t]{8}{*}{0.006} & $11.3 \mathrm{~b}$ \\
\hline & P. bourgaei & 3 & 0 & 3.423 & 0.275 & 0.476 & 3.200 & & $20.0 \mathrm{a}$ \\
\hline & $\begin{array}{l}P . \quad \text { grandiflora } \\
\text { var. grandiflora }\end{array}$ & 3 & 0 & 0.293 & 0.123 & 0.214 & 0.180 & & $2.7 \mathrm{c}$ \\
\hline & P. leucophracta & 3 & 0 & 1.510 & 0.637 & 1.103 & 0.960 & & $12.0 \mathrm{~b}$ \\
\hline & P. lycia & 3 & 0 & 1.223 & 0.173 & 0.300 & 1.210 & & $12.7 b$ \\
\hline & P. nissolii & 3 & 0 & 1.553 & 0.359 & 0.622 & 1.840 & & $14.0 \mathrm{~b}$ \\
\hline & $\begin{array}{l}P . \text { pungens var. } \\
\text { pungens }\end{array}$ & 3 & 0 & 0.443 & 0.111 & 0.191 & 0.500 & & $4.3 \mathrm{c}$ \\
\hline & P. samia & 3 & 0 & 9.963 & 0.315 & 0.546 & 9.710 & & $23.0 \mathrm{a}$ \\
\hline Component & Taxa & $\mathbf{N}$ & $\mathbf{N}^{*}$ & Mean & SEMean & StDev & Median & $\mathbf{P}$ & Rank \\
\hline \multirow{8}{*}{ 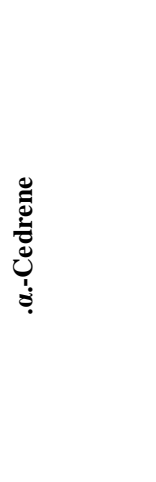 } & P. armeniaca & 3 & 0 & 0.000000 & 0.000000 & 0.000000 & 0.000000 & \multirow[t]{8}{*}{0.006} & $9.0 \mathrm{~b}$ \\
\hline & P. bourgaei & 3 & 0 & 0.183 & 0.183 & 0.318 & 0.000 & & $13.0 \mathrm{~b}$ \\
\hline & $\begin{array}{l}P . \quad \text { grandiflora } \\
\text { var. grandiflora }\end{array}$ & 3 & 0 & 24.40 & 2.71 & 4.69 & 25.92 & & $23.0 \mathrm{a}$ \\
\hline & P. leucophracta & 3 & 0 & 0.000000 & 0.000000 & 0.000000 & 0.000000 & & $9.0 \mathrm{~b}$ \\
\hline & P. lycia & 3 & 0 & 0.3533 & 0.0841 & 0.1457 & 0.4000 & & $19.0 \mathrm{a}$ \\
\hline & P. nissolii & 3 & 0 & 0.000000 & 0.000000 & 0.000000 & 0.000000 & & $9.0 \mathrm{~b}$ \\
\hline & $\begin{array}{l}P . \text { pungens var. } \\
\text { pungens }\end{array}$ & 3 & 0 & 0.000000 & 0.000000 & 0.000000 & 0.000000 & & $9.0 \mathrm{~b}$ \\
\hline & P. samia & 3 & 0 & 0.000000 & 0.000000 & 0.000000 & 0.000000 & & $9.0 \mathrm{~b}$ \\
\hline Component & Taxa & $\mathbf{N}$ & $\mathbf{N *}$ & Mean & SEMean & StDev & Median & $\mathbf{P}$ & Rank \\
\hline \multirow{7}{*}{ 窇 } & P. armeniaca & 3 & 0 & 14.64 & 1.57 & 2.71 & 15.73 & \multirow[t]{7}{*}{0.009} & $14.3 \mathrm{bc}$ \\
\hline & P. bourgaei & 3 & 0 & 19.17 & 2.25 & 3.89 & 20.80 & & $18.3 \mathrm{ab}$ \\
\hline & $\begin{array}{l}\text { P. } \text { grandiflora } \\
\text { var. grandiflora }\end{array}$ & 3 & 0 & 6.03 & 1.13 & 1.96 & 6.40 & & $5.0 \mathrm{de}$ \\
\hline & P. leucophracta & 3 & 0 & 21.630 & 0.756 & 1.309 & 22.320 & & $21.3 \mathrm{a}$ \\
\hline & P. lycia & 3 & 0 & 17.31 & 3.76 & 6.52 & 17.63 & & $16.7 \mathrm{ab}$ \\
\hline & P. nissolii & 3 & 0 & 11.383 & 0.617 & 1.069 & 11.280 & & $9.0 \mathrm{~cd}$ \\
\hline & $\begin{array}{l}P . \text { pungens var. } \\
\text { pungens }\end{array}$ & 3 & 0 & 0.930 & 0.236 & 0.410 & 0.740 & & $2.0 \mathrm{e}$ \\
\hline
\end{tabular}




\begin{tabular}{|c|c|c|c|c|c|c|c|c|c|}
\hline & P. samia & 3 & 0 & 14.247 & 0.477 & 0.826 & 13.790 & & $13.3 b c$ \\
\hline Component & Taxa & $\mathbf{N}$ & $\mathbf{N}^{*}$ & Mean & SEMean & StDev & Median & $\mathbf{P}$ & Rank \\
\hline \multirow{8}{*}{ 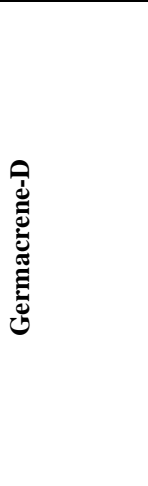 } & P. armeniaca & 3 & 0 & 25.23 & 1.09 & 1.89 & 25.03 & \multirow[t]{8}{*}{0.004} & $23.0 \mathrm{a}$ \\
\hline & P. bourgaei & 3 & 0 & 12.91 & 1.16 & 2.00 & 12.41 & & $13.3 \mathrm{c}$ \\
\hline & $\begin{array}{l}P . \quad \text { grandiflora } \\
\text { var. grandiflora }\end{array}$ & 3 & 0 & 4.857 & 0.433 & 0.751 & 5.170 & & $2.0 \mathrm{e}$ \\
\hline & P. leucophracta & 3 & 0 & 7.417 & 0.538 & 0.931 & 7.470 & & $5.7 \mathrm{de}$ \\
\hline & P. lycia & 3 & 0 & 16.43 & 2.95 & 5.10 & 15.66 & & $16.0 \mathrm{bc}$ \\
\hline & P. nissolii & 3 & 0 & 14.48 & 3.17 & 5.49 & 12.27 & & $13.7 \mathrm{c}$ \\
\hline & $\begin{array}{l}P . \text { pungens var. } \\
\text { pungens }\end{array}$ & 3 & 0 & 8.623 & 0.623 & 1.080 & 8.250 & & $7.3 \mathrm{~d}$ \\
\hline & P. samia & 3 & 0 & 21.12 & 1.31 & 2.27 & 21.01 & & $19.0 \mathrm{ab}$ \\
\hline Component & Taxa & $\mathbf{N}$ & $\mathbf{N}^{*}$ & Mean & SEMean & StDev & Median & $\mathbf{P}$ & Rank \\
\hline \multirow{8}{*}{ 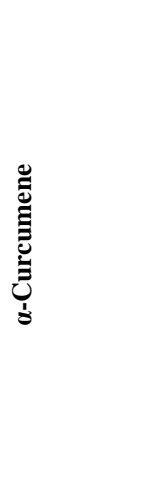 } & P. armeniaca & 3 & 0 & 0.000000 & 0.000000 & 0.000000 & 0.000000 & \multirow[t]{8}{*}{0.002} & $11.0 \mathrm{~b}$ \\
\hline & P. bourgaei & 3 & 0 & 0.000000 & 0.000000 & 0.000000 & 0.000000 & & $11.0 \mathrm{~b}$ \\
\hline & $\begin{array}{l}P . \quad \text { grandiflora } \\
\text { var. } \text { grandiflora }\end{array}$ & 3 & 0 & 13.040 & 0.575 & 0.995 & 13.240 & & $23.0 \mathrm{a}$ \\
\hline & P. leucophracta & 3 & 0 & 0.000000 & 0.000000 & 0.000000 & 0.000000 & & $11.0 \mathrm{~b}$ \\
\hline & P. lycia & 3 & 0 & 0.000000 & 0.000000 & 0.000000 & 0.000000 & & $11.0 \mathrm{~b}$ \\
\hline & P. nissolii & 3 & 0 & 0.000000 & 0.000000 & 0.000000 & 0.000000 & & $11.0 \mathrm{~b}$ \\
\hline & $\begin{array}{l}\text { P. pungens var. } \\
\text { pungens }\end{array}$ & 3 & 0 & 0.000000 & 0.000000 & 0.000000 & 0.000000 & & $11.0 \mathrm{~b}$ \\
\hline & P. samia & 3 & 0 & 0.000000 & 0.000000 & 0.000000 & 0.000000 & & $11.0 \mathrm{~b}$ \\
\hline
\end{tabular}

*a,b,c,d,e,f show differences between Phlomis taxa.

There was no statistically significant difference between $P$. pungens and P.armeniaca for vinly amly carnibol compound ratios, while there was a statistically significant difference between $P$. pungens and $P$. bourgaei, $P$. grandiflora, $P$. leucophracta, $P$. nissolii and $P$. samia. While there was no statistically significant different between $P$. grandiflora and $P$. samia. There was a statistically significant difference between $P$. grandiflora and P.armeniaca, $P$. bourgaei, $P$. leucophracta, $P$. lycia, $P$. nissolii and $P$. pungens samia.

There was no statistically significant difference between P. leucophracta, P. nissolii and $P$. samia for Limonene compound ratios according to the ordinal method, however there was a statistically significant difference between these three species and $P$. armeniaca, P. bourgaei, P. grandiflora, P. lycia, P. nissolii and P. pungens. Also there was no statistically significant difference between $P$. armeniaca, $P$. bourgaei, $P$. grandiflora, $P$. lycia, $P$. nissolii and $P$. pungens.

While there was no statistically significant difference between $P$. bourgaei and $P$. leucophracta for $\alpha$-cubebene compound ratios according to the ordinal method, there was a statistically significant difference between these two species and $P$. armeniaca, $P$. grandiflora, $P$. lycia, $P$. nissolii, $P$. pungens and $P$. samia. While there was no statistically significant difference between $P$. grandiflora and $P$. nissolii, there was a statistically significant difference between these two species and $P$. armeniaca, $P$. bourgaei, $P$. leucophracta, $P$. lycia, $P$. pungens and $P$. samia. 
There was a statistically significant difference between these two species and $P$. armeniaca, $P$. grandiflora, $P$. leucophracta, $P$. lycia, $P$. nissolii and $P$. pungens, however, there was no statistically significant difference between $P$. bourgaei and $P$. samia for $\alpha$-cubebene compound ratios according to the ordinal method, while there was no statistically significant difference between P. armeniaca, P. leucophracta, P. lycia and $P$. nissolii, while there was a statistically significant difference between these species and $P$. bourgaei, $P$. grandiflora, $P$. pungens and $P$. samia. Also there was no statistically significant difference between $P$. grandiflora and $P$. pungens.

While there was no statistically significant difference between $P$. grandiflora and $P$. lycia for $\alpha$-cedrene compound ratios according to the ordinal method, there was a statistically significant difference between these two species and $P$. armeniaca, P.bourgaei, P. leucophracta, P. nissolii, P. pungens and P. samia. There was no statistically significant difference between $P$. armeniaca, P.bourgaei, P. leucophracta, P. nissolii, $P$. pungens and P. samia.

There was no statistically significant difference between P.bourgaei, P. leucophracta and $P$. lycia for $\beta$-caryophyllene compound ratios according to the ordinal method, while there was a statistically significant difference between these species and $P$. armeniaca, $P$. grandiflora, $P$. nissolii, $P$. pungens and $P$. samia. While there was no statistically significant difference between $P$. grandiflora and $P$. pungens, there was a statistically significant difference between these two species and $P$. armeniaca, P.bourgaei, P. leucophracta, P. lycia, P. nissolii and P. samia

There was no statistically significant difference between, $P$. armeniaca and $P$. samia for germacrene-D compound ratios according to the ordinal method. There was a statistically significant difference between $P$. armeniaca and P.bourgaei, $P$. grandiflora, $P$. leucophracta, $P$. lycia, $P$. nissolii, $P$. pungens and $P$. samia. While there was no statistically significant difference between P.bourgaei and $P$. nissolii and $P$. lycia, statistically significant difference between P.bourgaei and $P$. nissolii and $P$. armeniaca, $P$. grandiflora, $P$. leucophracta, $P$. pungens and $P$. samia While there is not statistically significant difference between $P$. grandiflora and $P$. leucophracta, there was a statistically significant difference between $P$. grandiflora and $P$. armeniaca, P.bourgaei, P. lycia, $P$. nissolii, $P$. pungens and $P$. samia

There was a statistically significant difference between $P$. grandiflora and $P$. armeniaca, P.bourgaei, P. leucophracta, P. lycia, P. nissolii, P. pungens and P. samia for $\alpha$-curcumene component ratios per the ordinal method. There was no statistically significant difference between $P$. armeniaca, P.bourgaei, P. leucophracta, P. lycia, $P$. nissolii, $P$. pungens and $P$. samia.

Differences between the essential compounds of $\beta$-caryophyllene and germacrene-D were statistically important per the Friedman test in respect to volatile components by rates. The multi comparison Bonferroni-Dunn test was applied to determine the difference between the medians and found that there was a statistically significant difference between the pre bloom period, bloom period and post-emergence period for $\beta$-caryophyllene compound ratios according to an ordinal method. There was no statistically significant difference between the pre bloom period, bloom period and postemergence period for germacrene-D component ratios according to an ordinal method (Table 4). 
Table 4. Friedman testi results according to vegetation periods

\begin{tabular}{|c|c|c|c|c|c|c|c|c|c|}
\hline Component & Period & $\mathbf{N}$ & $\mathbf{N}^{*}$ & Mean & SEMean & StDev & Median & $\mathbf{P}$ & Rank \\
\hline \multirow{3}{*}{ a } & Before flowering & 8 & 0 & 13.76 & 2.51 & 7.10 & 14.76 & \multirow{3}{*}{0.001} & $17.0 \mathrm{~b}$ \\
\hline & Flowering & 8 & 0 & 15.03 & 2.83 & 7.99 & 15.91 & & $23.0 \mathrm{a}$ \\
\hline & After flowering & 8 & 0 & 10.71 & 2.16 & 6.12 & 11.09 & & $8.0 \mathrm{c}$ \\
\hline Component & Period & $\mathbf{N}$ & $\mathbf{N}^{*}$ & Mean & SEMean & StDev & Median & $\mathbf{P}$ & Rank \\
\hline \multirow{3}{*}{ 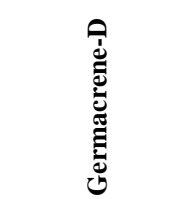 } & Before flowering & 8 & 0 & 12.88 & 2.44 & 6.89 & 12.34 & \multirow{3}{*}{0.010} & $12.0 \mathrm{~b}$ \\
\hline & Flowering & 8 & 0 & 16.47 & 2.84 & 8.03 & 17.93 & & $23.0 \mathrm{a}$ \\
\hline & After flowering & 8 & 0 & 12.31 & 2.31 & 6.52 & 10.82 & & $13.0 \mathrm{~b}$ \\
\hline
\end{tabular}

*a,b,c show differences between vegetation periods

\section{Discussion and Conclusion}

Yaşar et al. (2010) determined 12 volatile components for P. armeniaca. The main components were germakren-D (35.68\%), $\beta$-caryophyllene $(18.08 \%)$, caryophyllene oksit $(13.35 \%),(\mathrm{E})-\beta$-farnesene $(7.24 \%)$ and hexahydrofarnesyl aseton $(6.99 \%)$. They also determined that germakren-D was the dominant distinguishing compound of $P$. armeniaca. The results supported our thesis. Germakren-D was determined as dominant distinguishing compound.

Sixty-two volatile components of Phlomis bourgaei were found thru SPME analysis and these components of $P$. bourgaei had the following ratios: $\alpha$-cubebene (16.04\%), $\beta$ caryophyllene (21.98\%) and germakren-D (15.12\%). Sarkurkcu et al. (2013) determined the chemical compounds of volatile oils of $P$. bourgaei by hydro distillation and examine anti-toxicant potentials. The main components were: $\beta$-caryophyllene (37.37\%), (Z)- $\beta$-farnesene (15.88\%) and germakren-D (10.97\%). Baser et al. (2008) determined the components of $P$. bourgaei as germakren-D (11.3\%) and $\beta$ caryophyllene $(112 \%)$. $\beta$-caryophyllene and germakren-D were the main compounds. These findings support our research results. The only difference was that we determined that the $\alpha$-cubebene compound was the dominant compound.

In our research $\alpha$-pinene (26.40\%), $\alpha$-cedrene (28.15\%) and $\alpha$-curcumene (13.92\%) were volatile components of Phlomis grandiflora var. grandiflora among 60 components as determined by SPME analysis. Celik et al. (2005) determined components of $P$. grandiflora var. grandiflora as germakren-D (\%45.4\%), $\beta$ caryophyllene (22.8\%) and bicycle germakren (4.9\%). Demirci et al. (2008found that $\beta$ eudesmol (42\%) and $\alpha$-eudesmol (16\%), which are oxygenic sesquiterpenes as the most important compounds of $P$. grandiflora var. grandiflora. Ozcan et al. (2009) determined 32 components that represent $92.1 \%$ oil obtained from flowers. They determined the essential components as $\beta$-eudesmol (61.48\%), $\beta$-curcumene $(5.81 \%), \mathrm{E}-\beta$-farnesene (2.35\%), $\alpha$-zingiberene $(2.18 \%)$ and $\alpha$-cedrene (1.94\%). They found 39 compounds that represent $87.7 \%$ of oil obtained from leaves. Volatile components were $\beta$-eudesmol $(62.04 \%), \beta$-curcumene $(3.43 \%), \alpha$-curcumene $(2.20 \%)$ and linalool $(2.03 \%)$. It has been characterized that there are high percentages of $\beta$-eudesmol in both oils. The results of our study differ from the study of Celik et al. (2005). Meanwhile, Ozcan et al. (2009) found $\alpha$-cedrene and $\alpha$-curcumene as the main compounds in their studies. This 
result supports our study. In other research, $\alpha$-pinene has been found as the dominant component.

Seventy volatile components of Phlomis leucophracta were determined by SPME analysis and the essential compounds were (E)-2-hexenal (8.74\%), limonene $(14.56 \%)$ and $\beta$-caryophyllene (22.45\%). Celik et al. (2005) found $\beta$-caryophyllene $(20.2 \%), \alpha-$ pinen $(19.2 \%)$ and limonene $(11 \%)$ in P. leucophracta. $\beta$-caryophyllene and limonene were the volatile components. The result of this research supports our study. In other studies, (E)-2-hexenal was among the main component.

Sixty-two volatile components of Phlomis lycia were determined with SPME analysis and the main components of $P$. lycia were Limonene (17.68\%), $\beta$ caryophyllene $(23.66 \%)$ and germacrene-D (21.88\%).

Fifty-three volatile components of Phlomis nissolii were determined with SPME analysis and the main components of $P$. nissolii were limonene (23.75\%), $\beta$ caryophyllene (12.50\%) and germacrene-D (20.73\%) Kirimer et al. (2006) found 18 compounds thru GC/MS analysis. They found that the main components were germacrene-D (33.9\%), bicycle germacrene (15.3\%) and (Z)- $\beta$ - farnesene $(10.7 \%)$. Their results differ from our study. However, germacrene-D was found as a main component in both studies.

Seventy volatile compounds of Phlomis pungens var. pungens were determined and the main compounds were (E)-2-hexenal (17.60\%), vinly amyl carbinol (18.44\%) and germacrene-D (9.84\%). Masoudi et al. determined 24 compounds in Iran for P. pungens var. pungens thru GC/MS analysis. The volatile components included bicycle germacrene $(14.1 \%), \alpha$-pinen $(13.5 \%)$ and $(\mathrm{E})$ - $\beta$-farnese $(13.5 \%)$. These results differ from our study.

A total of 64 volatile components of Phlomis samia were determined by the SPME analysis. The main components were $\alpha$-copaene $(10.59 \%), \beta$-caryophyllene $(15.20 \%)$ and germacrene-D (23.44\%). Aligiannis et al. (2004) determined 72 components of upper sections of P.samia which grow in Greece and was represented at $67.4 \%$ by $\alpha-$ pinen, limonen, $\beta$ - caryophyllene, linaol, (E)- $\beta$-farnesene, germacrene -D, (Z)- $\gamma$ bisabolene and cis- $\beta$-ocimene. Demirci et al. (2006) determined germacrene -D (33.8\%) and $\beta$ - caryophyllene (6.4\%) as the main components of $P$. samia with GC/MS analysis. The above mentioned results support our results. Additionally, the $\alpha$-copaene compound was found.

The result of Kruskal-Wallis test found; 2-butenal, n-pentenal, trans-3-penten-2-one, 3-methyl-1-butanol, n-hexanal, (E)-2-hexenal, cis-hex-2-en-1-ol, hexanol <n->, amyl methyl ketone, n-heptanal, 2,4-hexadienal, $\alpha$-thujene, $\alpha$-pinene, (E)-2-heptenal, sabinene, . $\beta$-phellandrene, $\beta$-pinene, amyl vinyl ketone, vinly amly carbinol, 6 -methyl5-hepten-2-one, $\quad \beta$-myrcene, $\quad \mathrm{n}$-octanal, $\alpha$-phellandrene, 2,4-heptandienal, $\quad \mathrm{p}$ dichlorobenzene, $\alpha$-terpinene, $\mathrm{p}$-cymene, limonene, (E)-3-octen-2-one, cis-ocimene, 2 octenal, $\gamma$-terpinene, $\gamma$-terpinene, octanol, $\alpha$-terpinolene, dimethylstyrene $<\alpha$-para- $>, 2$ nonanone, methyl benzoate, n-nonanal, 2-hendecanone, $\alpha$-campholene aldehyde, transalloocimene, trans-2-nonenal, pinocarvone, (E)-2-nonenal, (E)-2-nonenal, n-decanal, (E)-2-decenal, $\alpha$-cubebene, $\alpha$-copaene, (-)-. $\beta$-elemene, sesquithujene <7-epi->, $\alpha$ gurjunene, $\alpha$-cedrene, $\beta$-caryophyllene, $\gamma$--elemene, $\alpha$-bergamotene, $(\mathrm{E})$ - $\beta$-farnesene, farnesol, epi-bicyclosesquiphellandrene, germacrene-D, ionone, $\alpha$-curcumene, bicyclogermacrene, zingiberene, $\alpha$-muurolene, $\beta$-muurolene, cedr- 8 -e,.$\gamma$-cadinene, $\delta$ cadinene, $\beta$-sesquiphellandrene, (-)-caryophyllene oxide, p-menthane, 2,3-dibromo-8- 
phenyl, androstan-17-one ve 3-ethyl-3-hydroxy and nonadecane components, and the differences between medians of Phlomis species were statistically significant.

The differences between medians of vegetation periods were statistically significant for hexanol <n->, n-heptanal, $\beta$-caryophyllene and germacrene-D components by Friedman test.

As a result, it is significantly important to reap samples of Phlomis taxa in the bloom period in respect to volatile compound productivity. These results are important in providing guidance for local growers and traders to reduce untimely plant picking and to reduce economic losses.

The leaves and flowers of Phlomis are used for several purposes, from making products more appetizing, as an anti-allergic, as a diuretic, for diarrhea preventive, against stomach aches, to relieve pain, anti-diabetic, and for herbal teas and tonics. Additionally, taxa that have yellowish flowers are important for food coloring. As such, research and further studies should be pursued in order to investigate how various plants can be used as a raw material for medicine or as food and cosmetic products. Importantly, future research will promote further application.

Acknowledgments. This study was a part of $\mathrm{PhD}$ thesis of first author Ayse Gul SARIKAYA. We express our sincere appreciation to Suleyman Demirel University for their financial support by project which numbered as 3360-D2-12.

\section{REFERENCES}

[1] Aligiannis, N., Kalpoutzakis, E., Kyriakopoulou, I., Mitaku, S., Chinou, I. B. (2004): Essential oils of Phlomis species growing in Greece: chemical composition and antimicrobial activity. - Flavour and Fragrance Journal 19: 320-324.

[2] Baser, K.H.C., Demirci, B., Dadandi, M.Y. (2008): Comparative Essential Oil Composition of the Natural Hybrid Phlomis $x$ vuralii Dadandı (Lamiaceae) and its Parents. - Journal of Essential Oil Research 20: 57-62.

[3] Baydar, H. (2009): Tibbi ve Aromatik Bitkileri Bilimi ve Teknolojisi. - Süleyman Demirel Üniversitesi Ziraat Fakültesi.

[4] Baytop, T. (1997): Türkçe Bitki Adları Sözlüğü. - TDK. Yayınları.

[5] Celik, S., Gokturk, R.S., Flamini, G., Cioni, P.L., Morelli, I. (2005): Essential oils of Phlomis leucophracta, Phlomis chimerae and Phlomis grandiflora var. grandiflora from Turkey. - Biochem Syst Eco 33: 617-623.

[6] Demirci, B., Dadandi, M.Y., Paper, D.H., Franz, G., Baser, K.H.C. (2003): Chemical composition of the essential oil of Phlomis linearis Boiss. \& Bal. and biological effects on the CAM-Assay: A safety evaluation. - Verlag der Zeitschriftfür Naturforschung 58: 826-829.

[7] Demirci, B., Baser, K.H.C., Dadandi, M.Y. (2006): Composition of The Essential Oils of Phlomis rigida Labill. and P. samia L. - Journal of Essential Oil Research 18: 328-331.

[8] Demirci, F., Guven, K., Demirci, B., Dadandi, M.Y., Baser, K.H.C. (2008): Antibacterial activity of two Phlomis essential oils against food pathogens. - Food Control 19: 11591164.

[9] Guner, A. (2012): Türkiye Bitkileri Listesi, Damarlı Bitkiler. - Nezahat Gökyiğit Botanik Bahçesi Yayınları.

[10] Harput, Ü.Ş., Calıs İ., Saracoglu, I., Donmez, A.A., Nagatsu, A. (2006): Secondary Metabolites from Phlomis syriaca and Their Antioxidant Activities. - Turkish Journal of Biology 30: 383-390 
[11] Huber-Morath, A. (1982): Phlomis L. in Flora of Turkey and East Aegean Islands. - In:. Davis, P.H. (ed.) Flora of Turkey and the East Aegean Islands, Edinburg University Press., Edinburg.

[12] Kirimer, N., Baser, K.H.C., Kurkcuoglu, M. (2006): Composition of the Essential Oil of Phlomis nissolii L. - Journal of Essential Oil Research 18: 600-601.

[13] Kumar, S.A. (2009): Plants-based Medicines in India. http://pib.nic.in/feature/feyr2000/fmay2000/f240520006.html. Accessed: 12.05. 2015

[14] Masoudi, S., Rustaiyan, A., Aberoomand, P. (2006): Composition of the Essential Oils of Cyclotrichium straussii (Bornm.) Rech. f. and Phlomis pungens Willd. from Iran. Journal of Essential Oil Research 18: 16-18.

[15] Mathiesen, C., Scheen, A. C., Lindqvist, C. (2011): Phylogeny and biogeography of the lamioid genus Phlomis (Lamiaceae). - The Board of Trustees of the Royal Botanic Gardens, Kew 66: 83-89.

[16] Ozcan, M.M., Tzakou, O., Couladis, M. (2009): Essential oil composition of the turpentine tree (Pistacia terebinthus L.) fruits growing wild in Turkey. - Food Chemistry 114: 282-285.

[17] Sarikurkcu, C., Ozer, M.S., Cakir, A., Eskici, M., Mete, E. (2013): GC/MS Evaluation and In Vitro Antioxidant Activity of Essential Oil and Solvent Extracts of an Endemic Plant Used as Folk Remedy in Turkey: Phlomis bourgaei Boiss. -Hindawi Publishing Corporation Evidence-Based Complementary and Alternative Medicine.

[18] Tan, A. (1992): Türkiye'de Bitkisel Çeşitlilik ve Bitki Genetik Kaynakları. - Anadolu Journal of AARI 2: 50-64.

[19] Vichy, S., Castellote, A. I., Pizzale, L., Conte, L. S., Buxaderas, S., Lopez-Tamames, E. (2003): Analysis of virgin olive oil volatile compounds by headspace solid-phase microextraction coupled to gas chromatography with mass spectrometric and flame ionization dedection. - Journal of Chromatography 983:19-33.

[20] Weiss, E.A. (1997): Essential Oil Crops. - The Journal of Agricultural Science 129: 121-123

[21] Yasar, S., Fakir, H., Erbas, S. (2010): Gas chromatographic (GC-GC/MS) analysis of essential oil of Phlomis armeniaca Willd. from Mediterranean region in Turkey. -Asian Journal of Chemistry 22: 2887-2890. 